\title{
Beweisverständnis im Mathematikstudium unterstützen: Vergleich unterschiedlicher Varianten der Strategie „Illustrieren am Beispiel““
}

\author{
Thomas Bauer (D) Eva Müller-Hill (D) - Silke Neuhaus-Eckhardt • \\ Stefanie Rach (D)
}

Eingegangen: 28. September 2020 / Angenommen: 15. September 2021 / Online publiziert: 13. Oktober 2021

(C) Der/die Autor(en) 2021

Zusammenfassung Das Lesen und Verstehen von Beweisen ist eine wichtige Aktivität in der wissenschaftlichen Disziplin Mathematik. In der Studieneingangsphase eines Mathematikstudiums stellt der lernförderliche Umgang mit Beweisen für die meisten Studierenden eine große Herausforderung dar. Beweise zu verstehen heißt nicht nur, einzelne Begründungsschritte im Beweis nachvollziehen, sondern beispielsweise auch, Hauptideen des Beweises identifizieren zu können. Welche individuellen Merkmale mit dem Beweisverständnis zusammenhängen und wie Studierende im Prozess des Beweisverstehens durch die spezifische Strategie der Beispielnutzung unterstützt werden können, steht im Zentrum der präsentierten Studie. 166 Studierenden mehrerer Analysis-Veranstaltungen wurde der Beweis eines Satzes über monotone Teilfolgen von reellwertigen Folgen vorgelegt. Die Studierenden wurden aufgefordert, diesen durchzulesen und Beispiele zu nutzen, um jeden einzelnen Beweisschritt zu illustrieren. Die Art des Illustrierens am Beispiel wurde in verschiedenen Varianten anhand zweier Bedingungen konkretisiert ( $2 \times 2$-Design): In der ersten Bedingung (,Lernaktivität“) wird unterschieden, ob die Studierenden ein zum gegebenen Beweis passendes Beispiel selbst konstruieren oder ob sie ein vorgegebenes Beispiel passiv nachvollziehen sollen. In der zweiten Bedingung (,Darstellungsform des Beispiels") wird das Beispiel entweder in symbolischer oder in graphischer Form genutzt. Die Ergebnisse zeigen, dass die schulischen Vorleistungen und insbesondere das inhaltliche Vorwissen das Beweisverständnis erwartungskonform stark prädizieren. Für einzelne Unterstützungsvarianten, konkretisiert in den

\footnotetext{
Thomas Bauer

Philipps-Universität Marburg, Marburg, Deutschland

Eva Müller-Hill

Universität Rostock, Rostock, Deutschland

Silke Neuhaus-Eckhardt $\cdot$ Stefanie Rach $(\bowtie)$

Otto-von-Guericke-Universität, Magdeburg, Deutschland

E-Mail: stefanie.rach@ovgu.de
} 
Bedingungen, konnten wir hingegen keine Vorteile für das individuelle Beweisverstehen ausmachen, dagegen geben die konkreten Bearbeitungsprozesse erhellende Einblicke in die Nutzung von Beispielen durch die Lernenden. Die Ergebnisse diskutieren wir in Bezug auf theoretische und praktische Implikationen für Lernprozesse im Mathematikstudium.

Schlüsselwörter Beweisverständnis · Illustrieren am Beispiel · Lernaktivität ·

Darstellungsform · Verstehensprozess

\title{
Supporting Proof Comprehension in University Mathematics Studies-Comparison of Different Options for the Strategy "Illustrating with Examples“
}

\begin{abstract}
Reading and understanding proofs is an essential activity in the scientific discipline of mathematics. In the introductory phase of a university mathematics program, it is a major challenge for students to engage with mathematical proofs in a way that supports learning. Understanding proofs does not only mean to comprehend individual steps of the proof, but it also means, for instance, to be able to identify the main ideas of the proof. The present study focuses on the question to which degree individual factors predict proof comprehension, and how students' proof comprehension processes can be supported through the specific strategy of using examples. The proof of the Monotone Subsequence Theorem was presented to 166 students from several Real Analysis courses. The students were prompted to read the proof and to use examples for illustrating every step of the proof. Groups of students were formed according to the way in which the use of examples is specified $(2 \times 2$-design): The first condition ('learning activity') differentiates between actively constructing an example to a given proof and passively retracing a given example. The second condition ('representation format') differentiates between symbolic vs. graphical presentation of the example. Our results show that final grades from high school and previous subject-matter knowledge strongly predict proof comprehension, as expected. By contrast, we could not give evidence for any advantages of the various conditions in the proof comprehension process, but we get illuminating insights into students' understanding processes. We discuss the results with an eye toward theoretical and practical implications.
\end{abstract}

Keywords Proof comprehension - Illustrating with examples · Learning activity · Representation formats $\cdot$ Process of understanding

\section{Einführung}

In den ersten Semestern eines Mathematikstudiums oder eines gymnasialen Lehramtsstudiums mit Fach Mathematik steht das Kennenlernen von Mathematik als wissenschaftliche Disziplin im Vordergrund, welche durch das mathematische Beweisen als eine reichhaltige und komplexe Aktivität geprägt ist. Dies betrifft zum Ersten den Forschungsprozess, in dem Vermutungen aufgestellt und getestet, ge- 
eignete Argumente und Argumentationsketten zu deren Stützung gefunden oder generiert und schließlich in Form von Theoremen und deren deduktiven Ableitungen aus bereits bewiesenen Resultaten zusammengeführt, organisiert, geprüft und kommuniziert werden, und zum Zweiten die akademische Lehre. Dozierende verwenden Beweise in den Lehrveranstaltungen u. a. zur Verifikation von Theoremen, zur Erklärung von Zusammenhängen sowie zur Kommunikation mathematischer Methoden und sozio-mathematischer Normen und Werte (Mejía-Ramos et al. 2012; Weber 2012). Studierende müssen solche Beweise in Vorlesungen und Lehrbüchern oder Skripten nachvollziehen, im Rahmen von Übungen und Prüfungen aber auch selbst generieren.

Es ist daher von besonderer Bedeutung, dass Studierende die in den Lehrveranstaltungen verwendeten Beweise verstehen, um sie entsprechend nutzen zu können. Aber nicht erst das Konstruieren eigener Beweise, sondern schon der Umgang mit gegebenen Beweisen stellt für viele Studierende eine große Hürde dar (vgl. z.B. Hodds et al. 2014; Selden 2012; Weber 2001). Vorliegende Untersuchungen und Ergebnisse zur Beweiskonstruktion und -validierung (Sommerhoff 2017) lassen die Annahme zu, dass das individuelle Vorwissen eine wichtige Rolle in Hinblick auf die Fragen spielt, welche Faktoren Beweisverständnis prädizieren und wie Studierende bestmöglich unterstützt werden können, mathematische Beweise zu verstehen. Grundsätzlich nimmt die Vielfalt an wirksamen Unterstützungsstrategien mit wachsender Erfahrung der Studierenden beim Lesen, Verstehen und Generieren von Beweisen zu. Von besonderem hochschuldidaktischen Interesse sind daher Strategien, die schon zu Beginn des Studiums, also bei Novizinnen und Novizen mit vergleichsweise geringem Erfahrungsstand, eine wirksame Unterstützung beim Verstehen von Beweisen liefern.

Mathematikdozierende gehen bei der Präsentation von Beweisen gelegentlich so vor, dass sie den Gang eines Beweises anhand der Durchführung an einem konkreten Beispiel illustrieren (vgl. Mali 2014; Mills 2014). So schlägt etwa Krantz (1999) explizit vor, den Beweis des Riemannschen Umordnungssatzes zunächst an einer konkreten Reihe durchzuführen, um sich auf die Kernidee des Beweises konzentrieren zu können. Eine Motivation für dieses Vorgehen kann die lange Tradition der Nutzung von Beispielen in der Lehre von Mathematik sein, die prominent unter anderem auf Felix Klein und dessen „Prinzip der Veranschaulichung“ zurückgeht; dieser nutzt etwa prototypische Beispiele, um abstrakte mathematische Begriffe und Konstruktionen in der Lehre veranschaulichen zu können (Klein 1908).

In welcher Weise die Nutzung der spezifischen Strategie „Illustrieren am Beispiel“ für Studierende mit Blick auf den Aufbau von Beweisverständnis hilfreich ist, ist jedoch nicht umfassend systematisch untersucht. Möglichkeiten der Unterstützung von Studierenden durch die Strategie des Illustrierens am Beispiel untersuchen wir in der hier vorgestellten Studie auf zwei verschiedenen Ebenen: (1) auf der Ebene der damit verbundenen Lernaktivität und (2) auf der Ebene der Darstellungsform des Beispiels. Mit Blick auf (1) unterscheiden wir eine stärker aktiv-konstruktive Unterstützung - die Studierenden werden angeregt, ein eigenständiges konkretisierendes Fallbeispiel zu gegebenen Beweisschritten zu konstruieren - von einer passiven Unterstützung (vgl. Chi 2009) - die Studierenden werden angeregt, zusammen mit dem Beweis ein gegebenes Beispiel zu lesen. Im Rahmen von (2) gehen wir der 
Frage nach, ob eine graphische oder eine symbolische Repräsentation des mathematischen Begriffs ,reellwertige Folge“ im Falle des vorgegebenen konkretisierenden Fallbeispiels das Beweisverständnis stärker unterstützt (vgl. Gagatsis et al. 2006).

Das Konstrukt „Beweisverständnis“ ist dabei selbst facettenreich und komplex. Unter den möglichen Perspektiven, die man bei einer didaktisch motivierten empirischen Untersuchung darauf einnehmen kann, wählen wir hier eine im weiteren Sinne lernpsychologische. Wir fokussieren dabei auf die Aktivität des Lesens eines schriftlichen Beweises und auf einen niedrigschwelligen Transfer der einzelnen Beweisschritte auf ein konkretisierendes mathematisches Fallbeispiel. Als zugrundeliegendes operationalisiertes Modell für eine nachgängige Erhebung von Beweisverständnis verwenden wir das in der Literatur verbreitete Modell von Mejía-Ramos et al. (2012).

Im Forschungsstand präzisieren wir den Begriff „Beweisverständnis“, fassen vorliegende Ergebnisse zu möglichen Prädiktoren und ausgewählten Unterstützungsmaßnahmen zum Beweisverständnis zusammen und formulieren Forschungsfragen. Die empirische Studie mit 166 Fach- bzw. Lehramtsstudierenden aus mehreren Analysisveranstaltungen im 2. Semester, das zugehörige Design und die verwendeten Instrumente stellen wir im Anschluss dar. Abschließend diskutieren wir die Ergebnisse bezüglich theoretischer und praktischer Implikationen.

\section{Theoretische Grundlagen und Forschungsstand}

Der Begriff des mathematischen Beweises selbst wird in der Literatur in vielfältigen Lesarten verwendet. Während das Bedeutungsspektrum in der wissenschaftlichen Mathematik von Beweisen als formalen Ableitungen bis hin zu mehr oder weniger informellen, deduktiven Argumentationsketten reicht, die aber stärker formalisiert werden könnten, zeugt die einschlägige mathematikdidaktische Literatur von einem recht weiten Verständnis von mathematischen Beweisen als (nicht unbedingt streng deduktive) Argumentationsketten und umfasst auch präformale Beweise (der Begriff geht auf Semadeni (1974) und Kirsch (1979) zurück), operative Beweise (Müller und Wittmann 1988) und generische Beweise (z. B. Rowland 2002; für einen Überblick Mali 2014). Einen genaueren Überblick zum didaktischen Bedeutungsspektrum geben Biehler und Kempen (2016) und Brunner (2014).

Mathematische Beweise erfüllen dabei in der wissenschaftlichen Mathematik, aber auch beim Lehren und Lernen von Mathematik unterschiedliche Funktionen, darunter Verifikation, Erklärung, Entdeckung, Systematisierung und Kommunikation (De Villiers 1990), Rechtfertigung von Definitionen oder axiomatischen Strukturen (Weber 2002) und Ermöglichung ästhetischer Erfahrung (Rav 1999). Insbesondere über die Erklärfunktion sind mathematische Beweise an das subjektive Verstehen gekoppelt: Ein erklärender Beweis macht der Rezipientin oder dem Rezipienten deutlich, warum das bewiesene Theorem gilt (vgl. Müller-Hill 2017). Der für unsere Studie ausgewählte Beweis stellt einen typischen Vertreter eines Beweises dar, wie er in einer Analysis-Vorlesung präsentiert werden würde. Es handelt sich um eine deduktive, semi-formalisierte Argumentationskette, die sowohl in der wissen- 
schaftlichen Mathematik als auch in der fachdidaktischen Sprechweise als Beweis bezeichnet werden würde.

Noch vielschichtiger als der Beweisbegriff selbst ist der Begriff des Beweisverständnisses. Wir gehen im Folgenden genauer auf mögliche Bedeutungen dieses Begriffs ein.

\subsection{Aspekte von vorliegendem Beweisverständnis vs. Strategien zum Beweisverstehen}

Die Bedeutung von „Beweisverständnis“ bzw. „Verstehen von Beweisen“ wird unter anderem im Rahmen von soziomathematischen und wissenschaftsphilosophischen Expertenstudien untersucht - hier werden sowohl Expertinnen und Experten bei ihrem mathematischen Tun (d.h. hier: Beweise lesen) beobachtet als auch nach ihrem Eigenverständnis dieses Tuns befragt. Demnach bedeutet Beweisverständnis weder, dass ein Beweis auswendig Wort für Wort wiedergegeben, noch, dass er selbst gefunden werden können muss (vgl. z. B. Heintz 2000; Müller-Hill 2011). Stattdessen sollen etwa jeder einzelne Beweisschritt sowie die übergreifende Beweisidee nachvollzogen und ggf. auf neue Situationen angewendet werden können. Ältere Arbeiten zur Messung von Beweisverständnis bei Mathematiklernenden begrenzen das Bedeutungsspektrum von Beweisverständnis in ähnlicher Weise (vgl. Pracht 1979; aber z. B. auch Conradie und Frith 2000).

Auch in der neueren lernpsychologischen Literatur sind Studien (z.B. Hodds et al. 2014; Mejía-Ramos et al. 2012; Weber 2015; Weber und Mejía-Ramos 2011) zu finden, in denen Beweisverständnis fokussiert wird. Dabei werden zwei unterschiedliche Blickrichtungen eingenommen: Zum einen werden auch hier Expertenstudien mit ähnlichen Fragestellungen durchgeführt. Zum anderen wird die Frage fokussiert, inwiefern Studierende über Beweisverständnis verfügen, und Operationalisierungsmodelle entwickelt, um Beweisverständnis zu messen. Solche Operationalisierungsmodelle und Expertenstudien liefern mehrheitlich Listen mit Aspekten von Beweisverständnis (in Form von Indikatoren; z. B. Mejía-Ramos et al. 2012) bzw. im Falle der Expertenstudien auch Strategien zum Beweisverstehen (z. B. Weber 2015). Dem vielschichtigen Konstrukt „Beweisverständnis“ werden Aspekt- und Strategielisten allerdings noch nicht unbedingt gerecht.

Auch stellt sich die Frage, inwieweit Studien, die wesentlich auf Expertenbefragungen von professionellen Mathematikerinnen und Mathematikern beruhen, den Anspruch erheben dürfen, der Novizenrolle von Mathematiklernenden gerecht werden zu können. So stellen eine Reihe von Arbeiten und Modellen Aspekte oder Strategien eines niedrigschwelligen „Verstehens-von-Beweisen“ recht unvermittelt neben solche eines deutlich anspruchsvolleren „Verstehens-durch-Beweise“. Beim Verstehen-von-Beweisen wird der gegebene Beweis zeilenweise und mit Fokus auf das Nachvollziehen seiner inferentiellen Struktur erfasst (dies meint die Identifikation von Voraussetzungen und Behauptungen sowie die Einsicht darin, wie die einzelnen Beweiszeilen logisch zusammenhängen). Unter Verstehen-durch-Beweise fassen wir dagegen ein gedankliches Durchdringen des Beweisganges, bei dem die inferentielle Struktur über das reine Nachvollziehen hinaus sinnhaft interpretiert und bewertet wird. Hierbei werden kritische Stellen wie zentrale Begriffsdefinitionen und 
entscheidende Ableitungsschritte identifiziert und beispielsweise von austauschbaren, technischen Details unterschieden. Erst auf der Basis eines solchen Verstehensdurch-Beweise erkennt die Rezipientin oder der Rezipient eines Beweises, warum das bewiesene Theorem gilt und welche im Beweis benutzten Methoden und Techniken über den gegebenen Beweis in seiner konkreten Ausführung hinausweisen. Aus Sicht von Expertinnen und Experten ist gerade ein Verstehen-durch-Beweise im obigen Sinne zentral (vgl. Müller-Hill 2011), während für Mathematiklernende zunächst eher ein Verstehen-von-Beweisen im Vordergrund steht. Das genauere Zusammenspiel von Verstehen-von-Beweisen und Verstehen-durch-Beweise ist bisher jedoch nicht hinreichend untersucht.

\subsection{Illustrieren am Beispiel als Strategie zum Beweisverstehen}

Wir möchten in dieser Arbeit eine dritte Blickrichtung einnehmen. Mit Blick auf „Illustrieren am Beispiel“" wollen wir explizit der Frage nachgehen, wie das Illustrieren am Beispiel als Strategie zum Beweisverstehen bei Novizinnen und Novizen funktionieren kann. Dabei wollen wir insbesondere weitergehend betrachten, unter welchen inhalts- und prozessspezifischen Bedingungen diese Strategie den Verstehensprozess fördern kann. Beispiele verstehen wir dabei nach Zaslavsky (2019, S. 2) als „objects satisfying certain conditions“ (vgl. Watson und Mason 2005) im Sinne von konkreten Repräsentanten für ein mathematisches Prinzip, ein mathematisches Konzept, eine mathematische Idee oder eine bestimmte mathematische Eigenschaft. Weiterhin muss das Objekt oder der Repräsentant von Lehrenden oder Lernenden, insbesondere auch Novizinnen und Novizen, als Beispiel anerkannt sein (Zaslavsky 2019).

Wir konzentrieren uns in der hier vorgestellten Studie auf die Frage, wie konkrete Aktivitäten von Studierenden im Rahmen des Lesens und zeilenweisen Nachvollziehens eines deduktiven Beweises, also insbesondere schon beim Verstehen-vonBeweisen, gestaltet werden sollten, um der Strategie des Illustrierens am Beispiel zur Wirksamkeit zu verhelfen. Dies ist, wie weiter oben bereits beschrieben, unter anderem auch durch die Beobachtung motiviert, dass Mathematiklehrende an Hochschulen „Illustrieren am Beispiel“ für eine sehr wirksame Strategie zur Unterstützung beim Beweisverstehen für Studierende halten und dieses entsprechend praktizieren, etwa durch Beispiele, die parallel zur Präsentation eines Beweises zur Instanziierung von Aussagen innerhalb des Beweises vorgegeben werden (,,instantiation of a subclaim“, Mills 2014, S. 115).

Darüber hinaus ist dieser Blick lerntheoretisch motivierbar. So schlagen Ahmadpour et al. (2019) beispielsweise ein theorie- und datenbasiertes, dynamisches Modell für Beweisverstehen vor, welches zwischen unterschiedlichen Formen semantischen und syntaktischen Verstehens unterscheidet. Unter Rückgriff auf die lerntheoretische Tradition nach Piaget sowie Arbeiten von Semadeni (1974) weisen sie dabei für semantisches Verstehen, welches insbesondere das Verstehen von im Beweis verwendeten Prozeduren als allgemein anwendbare Prozeduren auf Objekte aus einer bestimmten Klasse umfasst, die Verankerung in beispielgebundenen Aktivitäten als grundlegend aus: Werden Beweise bzw. einzelne Beweisschritte im Sinne von allgemeinen Prozeduren gelesen und verstanden, so umfasst dies das Ver- 
stehen der Bedeutung der jeweiligen Prozedur als ,,a procedure that can be applied to any number to check that it satisfies the given statement" (Ahmadpour et al. 2019, S. 8). Mit Blick auf die dort betrachteten Daten, im konkreten Fall Bearbeitungen von jungen Mathematiklernenden (siebte und achte Klasse), werden dabei gerade Verstehensprozesse im Sinne des Verstehens-von-Beweisen untersucht. In der im vorliegenden Beitrag präsentierten Studie untersuchen wir, ob ein zeilenweises Anwenden der in einzelnen Teilschritten eines gegebenen formal-symbolischen Beweises relevanten allgemeinen Prozeduren (in unserem Fall ein allgemeines, induktives Auswahlverfahren von Teilfolgengliedern) auf konkrete Beispiele dabei hilft, den Beweis semantisch zu verstehen (also als einen ,formulated proof“ oder als einen ,,procedural proof“), und nicht nur als ein rein syntaktisches Argument auf symbolischer Ebene zu lesen (als einen „formulaic proof“).

Neben der mit den hier untersuchten Fragestellungen verbundenen Annahme, dass es von inhalts- und prozessspezifischen Bedingungen abhängt, ob ein im Rahmen einer Intervention eingebrachtes oder eingefordertes Beispiel förderlich für das Beweisverstehen sein kann, ist grundsätzlich zu beachten, dass individuelle Einflussfaktoren einen möglichen positiven Effekt des Illustrierens am Beispiel auf den Verstehensprozess überlagern. Auch kann ein solcher Effekt davon beeinflusst werden, dass durch die Beispielbetrachtung der gewohnte Lesefluss als Teil des individuellen Verstehensprozesses ad hoc unterbrochen wird. Diese möglicherweise relevanten Bedingungen und Faktoren werden in den nächsten Abschnitten vorgestellt und erläutert.

\subsection{Individuelle Einflussfaktoren auf den Verstehensprozess}

Theoretisch wird angenommen, dass individuelle Merkmale bzw. Ressourcen von Lernenden, wie Vorwissen und schulische Vorleistungen, direkt auf den Verstehensprozess von Beweisen wirken, und der Verstehensprozess dann das Verständnis als Ergebnis dieses Prozesses beeinflusst. In empirischen Studien wird der Verstehensprozess jedoch häufig nicht explizit analysiert, so dass zunächst ein direkter Effekt von individuellen Merkmalen auf das Beweisverständnis untersucht wird (siehe Abb. 1).

So scheinen etwa die schulischen Vorleistungen das Beweisverständnis zumindest im Bereich der Zahlentheorie zu prädizieren (Neuhaus und Rach 2019). Diese Annahme wird dadurch begründet, dass gerade die Gesamt-Abiturnote, die in vielen Arbeiten als Indikator für die allgemeine Schulleistung verwendet wird, einen Einblick in kognitive Fähigkeiten und auch Hinweise auf die Ausprägung nichtkognitiver Fähigkeiten, wie z. B. Lernbereitschaft, gibt (z. B. Trapmann et al. 2007), die das individuelle Beweisverstehen beeinflussen können. Andere individuelle Ressourcen wie z.B. motivationale Merkmale scheinen einen eher geringeren Einfluss auf das Beweisverständnis zu haben (Neuhaus und Rach 2019).

Inhaltliches Vorwissen, gerade konzeptionelles Wissen um die im Beweis vorkommenden Begriffe, scheint insbesondere bedeutend für den Verstehensprozess von Beweisen zu sein. Wenn beispielsweise Eigenschaften von Begriffen nicht bekannt sind, dann kann auch nicht verstanden werden, an welchen Stellen des Beweises diese zur Begründung herangezogen werden. Empirische Ergebnisse zum Zu- 


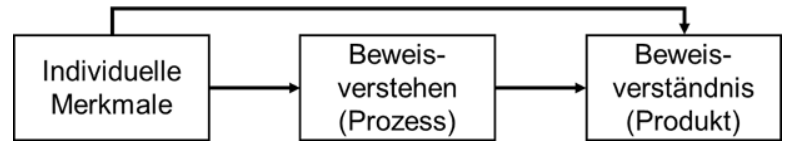

Abb. 1 Zusammenhang zwischen individuellen Merkmalen, dem Prozess des Beweisverstehens und dem Beweisverständnis als Produkt dieses Prozesses

sammenhang zwischen inhaltlichem Wissen und verschiedenen Komponenten des Beweisens stützen diese Annahme (Neuhaus und Rach 2019; Sommerhoff 2017).

\subsection{Bedingungen für das Illustrieren am Beispiel als Strategie zum Beweisverstehen}

In der bisherigen Forschung zu Unterstützungsmöglichkeiten für den Verstehensprozess von Beweisen wurde unter anderem das Leseverhalten unterschiedlicher Gruppen von Beweisrezipientinnen und -rezipienten analysiert. In Experten-Novizen-Vergleichen konnte ermittelt werden, dass Mathematikerinnen bzw. Mathematiker und erfolgreiche Studierende einen Beweis grundsätzlich unterschiedlich lesen und unterschiedliche Strategien nutzen im Vergleich zu Novizinnen und Novizen (z. B. Inglis und Alcock 2012). Die von Weber (2015) durch Beobachtungsstudien als hilfreich identifizierte Strategie „Illustrieren am Beispiel“ in dem Sinne, einen schwierigen Beweisabschnitt oder den gesamten Beweis anhand von Beispielen zu illustrieren und so besser nachvollziehen zu können, nutzen Mathematikerinnen und Mathematiker dabei häufiger als Novizinnen und Novizen (Wilkerson-Jerde und Wilensky 2011; vgl. auch Weber und Mejía-Ramos 2013).

Insbesondere nutzen Novizinnen und Novizen Beispiele beim Beweisverstehen kaum ,spontan“ im Sinne von Zaslavsky (2019), die mit Blick auf Mathematiklernende drei verschiedene Arten der Beispielnutzung unterscheidet: spontan, evoziert und reagierend. Spontan wird ein Beispiel genutzt, wenn es weder induziert noch verlangt wurde. Lernende wählen selbstständig ein Beispiel, um sich z.B. einen unklaren Beweisschritt zu verdeutlichen. Eine Beispielnutzung ist evoziert oder hervorgerufen, wenn z.B. in einer Aufgabe explizit verlangt wird, ein Beispiel zu verwenden. Sowohl bei der spontanen als auch bei der evozierten Beispielnutzung wählen jeweils die Lernenden ein Beispiel aus. In der dritten Kategorie wird dagegen ein Beispiel vorgegeben und die Lernenden reagieren darauf. Beispiele hierfür sind ausgearbeitete Lösungen oder von Dozierenden ausgewählte Beispiele, die ein Konzept verdeutlichen sollen.

Aufgrund unseres Fokus auf Studierende zu Beginn ihres Mathematikstudiums beschränken wir uns auf die Untersuchung eines möglichen positiven Effekts von evozierter und reagierender Beispielnutzung auf das Beweisverstehen.

\subsubsection{Lernaktivitäten: Evozierte Konstruktion versus passiv-reagierendes Nachvollziehen von Beispielen als prozessbezogene Bedingungen}

Die Unterscheidung von passiven, aktiven, konstruktiven und interaktiven Lernaktivitäten geht auf die Arbeiten von Chi (2009) zurück und lässt sich mit Blick auf 
das Lesen eines Textes wie folgt verdeutlichen: Beim passiven Lesen wird leise oder laut gelesen, ohne etwas anderes zu tun (Chi und Wylie 2014). Aktives Lesen bedeutet beispielsweise, bestimmte Textstellen zu unterstreichen, während konstruktives Lesen sich darin widerspiegelt, dass Notizen zu diesem Text erstellt werden. Auch das Bilden von Selbsterklärungen fällt unter eine konstruktive Lernaktivität, da neue Informationen zum Text entwickelt werden. Interaktives Lesen bedeutet beispielsweise, dass Fragen und Antworten zum Verständnis des Textes mit einem Partner bzw. einer Partnerin ausgetauscht werden. Empirische Studien belegen die Wirkweise der verschiedenen Lernaktivitäten (vgl. Chi und Wylie 2014), wobei die Lernaktivitäten im Folgenden aufsteigend nach ihrem erwarteten Erfolg notiert sind: passiv, aktiv, konstruktiv, interaktiv. Ufer (2015) weist insbesondere den positiven Einfluss von konstruktiven Lernaktivitäten auf den Studienerfolg in einem Mathematikstudium nach. In der Lösungsbeispielforschung wird darüber hinaus diskutiert, ob bestimmte Gruppen von Lernenden von bestimmten Lernaktivitäten insbesondere profitieren. Unter dem Begriff ,expertise reversal effect“ ist das Phänomen zu finden, dass Lernende mit geringerem Vorwissen von anderen Aktivitäten profitieren als Lernende mit höherem Vorwissen (Kalyuga et al. 2003). Beispielsweise zeigen Leppink et al. (2012) im Bereich Statistik, dass Lernende mit geringem Vorwissen vom Lernen aus Lösungsbeispielen profitieren, während Lernende mit höherem Vorwissen vom Formulieren von Argumenten, also einer konstruktiven Lernaktivität, eher profitieren.

In unserer Studie untersuchen wir Unterschiede zwischen passiven Lernaktivitäten und konstruktiven Lernaktivitäten zur Beispielnutzung beim Beweisverstehen. Passiv bedeutet dabei, dass ein geeignetes Beispiel vorgegeben ist und die Lernenden dieses Beispiel nachvollziehen sollen, was der reagierenden Beispielnutzung nach Zaslavsky (2019) entspricht. Konstruktiv bedeutet, dass die Lernenden selbst ein Beispiel - als über das vorgelegte Beweismaterial hinausgehende, neue Information - entwickeln sollen. Da den Studierenden weiterhin vorgegeben wird, dass sie ein Beispiel konstruieren müssen, handelt es sich hierbei um eine evozierte Beispielnutzung nach Zaslavsky (2019).

Iannone et al. (2011) stellen entgegen ihren Erwartungen überraschenderweise fest, dass bei Aktivitäten zur Illustration von relevanten Begriffsdefinitionen an Beispielen das aktive Generieren illustrierender Beispiele für das Lösen nachgeschalteter Beweisproduktionsaufgaben keine Vorteile gegenüber dem passiven Beispiellesen bringt. Mit Blick auf unsere Intervention, in der wir die etwas anders gelagerte Frage nach Auswirkungen des aktiven Generierens von Beispielen zur zeilenweisen Illustration eines gegebenen Beweises im Unterschied zum passiven Lesen entsprechender Beispiele auf das verständige Nachvollziehen des Beweises untersuchen, ziehen wir aus diesen Ergebnissen von Iannone et al. zwei Schlussfolgerungen. Zum einen haben die von den Probandinnen und Probanden in der Studie geforderten bzw. die ihnen vorgelegten Beispiele weniger die für den späteren Beweis wesentlichen Aspekte der vorkommenden Schlüsselbegriffe des Beweises illustriert, deren Verständnis dann für die Beweiskonstruktion nötig war. Tatsächlich lagen auch einige der Schwierigkeiten von Probandinnen und Probanden der aktiven Gruppe bei den speziellen Beispielkonstruktionen bereits auf der Ebene der algebraischen Manipulationen vor, so dass beispielsweise ein Rückschluss auf ursächli- 
che Schwierigkeiten für das Beweisverständnis auf konzeptioneller Ebene erschwert wird. Im Unterschied dazu und um die zusätzliche Schwierigkeit des Umgangs mit algebraischen Manipulationen zu vermeiden, müssen in der von uns vorgestellten Intervention weder bei der passiven noch bei der konstruktiven Beispielnutzung algebraische Manipulationen nachvollzogen bzw. vorgenommen werden. Zum anderen diskutieren Iannone et al. (2011) selbst als eine mögliche Erklärung für ihren unerwarteten Befund, dass einbettende Maßnahmen im Sinne von ,explicit instruction in how to generate examples" (ebd., S. 11) nötig sein könnten, damit das aktive Beispielgenerieren hinreichend wirksam werden kann: „this account suggests that the task [of example generation] per se is not effective, but rather that only certain approaches to tackling the task may be effective“" (ebd.). Ergänzend legen die Ergebnisse von Samkoff und Weber (2015) nahe, dass die Art des Umgangs mit einem gewählten Beispiel eine wesentliche Rolle dafür spielt, wie Beweisverstehen durch das Illustrieren am Beispiel unterstützt wird. Besonders wirksam sind demnach Beispiele, mit denen generisch umgegangen wird, also solcherart, dass die am Beispiel illustrierten Beweisschritte zwar auf Ebene der konkreten Repräsentanten durchgeführt werden, aber nur mit Rückgriff auf solche Eigenschaften, die diese konkreten Objekte als Charakteristika für eine ganze Klasse von Objekten aufweisen, für die der Beweis gilt (in diesem Sinne verwendet auch Balacheff (1988) den Begriff des generischen Beispiels). Im Rahmen unserer Intervention geben wir daher als eine indirekte Instruktion sowohl im konstruktiven als auch im passiven Fall zunächst ein zeilenweise illustrierendes, hilfreiches (vgl. Abschn. 4.6) Beispiel für den ersten Beweisabschnitt vor.

\subsubsection{Darstellungsformen: Graphische versus symbolische Darstellungen von Beispielen als inhaltsbezogene Bedingungen}

Weitere Forschungsergebnisse liegen mit Blick auf den Einfluss unterschiedlicher Darstellungen von Beweisen auf das Beweisverständnis vor, etwa zu strukturierten Beweise nach Leron (1983) oder zu sogenannten e-proofs nach Roy et al. (2010, 2017; vgl. für das Textverständnis im naturwissenschaftlichen Bereich Härtig et al. 2019). Aus mathematikdidaktischer Perspektive stellt sich hier die Frage, ob die Darstellungsform des gewählten Beispiels einen Einfluss auf das Beweisverstehen hat. Auch mit Blick auf die bereits angeführte Arbeit von Ahmadpour et al. (2019) läge eine positive Antwort auf diese Frage zumindest nahe, denn ein durch das Illustrieren am Beispiel potenziell unterstütztes semantisches Verstehen von Beweisen basiert unter anderem darauf, Analogien zwischen Handlungen und Operationen am konkreten Beispiel und allgemeinen Prozeduren im Rahmen der Beweisführung zu erkennen. Die Wahl der Darstellungsebene für das konkrete Beispiel hat offenbar Auswirkungen auf die daran ausführbaren Handlungen und Operationen und beeinflusst damit auch die Art des Erkennens der Beziehung zwischen Beispiel und allgemeinem Beweis(schritt). Aus Sicht der MultiMedia-Forschung (vgl. Schnotz und Bannert 2003) führt das Verwenden einer graphischen Darstellung neben einer symbolischen Darstellung nicht zwangsläufig zu einem besseren Verständnis, denn die weitere Darstellungsform kann zu extraneous cognitive load führen. Dieser negative Effekt tritt insbesondere dann auf, wenn die graphische Darstellungsform nicht 
zum mentalen Modell passt, welches durch die symbolische Darstellung aufgebaut wird.

Typischerweise sind Beispiele, die zum Beweisverstehen genutzt werden, konkrete mathematische Objekte, die unter einen für den Beweis wichtigen mathematischen Begriff fallen. Unsere Studie ist eingebettet in das mathematische Gebiet „Analysis“, weswegen wir hier vor allem auf wichtige mathematische Begriffe in diesem Gebiet eingehen. Ausgehend von den lernpsychologischen Theorien von Bruner (1971) stellen Übergänge zwischen enaktiven, ikonischen und symbolischen Repräsentationen des jeweiligen Begriffes ein wesentliches Element von Begriffsbildungsprozessen dar. Der Zielzustand eines flexiblen Verfügens über einen Begriff zeichnet sich durch ein reichhaltiges concept image aus (Tall und Vinner 1981), was sich performativ insbesondere dadurch zeigt, dass unterschiedliche Repräsentationen für unterschiedliche Problemstellungen passend gewählt werden, etwa mit Blick darauf, welche der den Begriff definierenden Eigenschaften besonders fokussiert werden sollen und welche Repräsentationsform dafür besonders hilfreich ist. In unserer Studie haben wir einen Beweis gewählt, in welchem sich ein Schlüsselbegriff besonders gut an Beispielen auf unterschiedlichen Repräsentationsebenen konkretisieren lässt (siehe Abschn. 4.4), und das so unterstützte konzeptionelle Verständnis im Sinne des concept image (Tall und Vinner 1981) auch eine mutmaßlich starke Unterstützung beim zeilenweisen Verstehen des Beweises liefern kann. Der Transfer auf das Beispiel ist dabei möglichst niedrigschwellig gewählt, er lässt sich quasi „durch Einsetzen“ erledigen (vgl. genauer die Beschreibung des Interventionsdesigns in Abschn. 4.4).

Der Übergang zur akademischen Mathematik mit einer gesteigerten Bedeutung von formalen Begriffen und symbolischen Darstellungen bereitet den Studierenden häufig große Schwierigkeiten (Clark und Lovric 2009; Tall 2008). Aus diesem Grund kann es hilfreich sein, wenn der Umgang mit symbolischen Repräsentationen durch das Aktivieren ikonischer Repräsentationen wie tabellarischen oder graphischen Darstellungen oder Prototypen unterstützt wird. Für die in der Analysis zentralen reellwertigen Abbildungen, worunter auch Folgen als Spezialfall betrachtet werden können, ist neben der symbolischen Repräsentationsform mit Hilfe einer Gleichung auch eine graphische Darstellung wichtig (Gagatsis et al. 2006; Ronda 2015). Beispiele für dieses Vorgehen sind in der Studie von Roh (2009) zu erkennen: Die untersuchten Studierenden nähern sich dem Konvergenzbegriff reellwertiger Folgen, indem sie anhand einer graphischen Darstellung verschiedene Beispiele und Gegenbeispiele von konvergenten Folgen untersuchen. Gerade im Bereich der Konvergenz von Folgen gibt es starke Hinweise, dass graphische Darstellungen den Lernprozess unterstützen (Ostsieker 2020). Empirische Ergebnisse unterstreichen aber auch, dass Lernende nur selten eigenständig und spontan verschiedene Darstellungsformen, vor allem graphische Darstellungen, nutzen (Acevedo Nistal et al. 2014). Auch in unserer Intervention ist der Begriff der Folge grundlegend, und wie schon mit Blick auf die Nutzung der Strategie „Illustrieren am Beispiel“ an sich scheint es mit Blick auf unsere Zielgruppe aus Novizinnen und Novizen ebenfalls passend, den Wechsel von der symbolischen Repräsentation des betrachteten Beweises in eine ikonische Darstellung des illustrierenden Beispiels vorzugeben bzw. zu evozieren. Wir vergleichen daher die Wirksamkeit von „Illustrieren am Beispiel““ 
anhand eines evozierten bzw. präsentierten symbolisch repräsentierten Beispiels mit der eines ikonisch repräsentierten Beispiels.

\section{Forschungsfragen}

Beweisaktivitäten spielen eine große Rolle in einem Mathematikstudium, stellen aber für viele Studierende ungewohnte Hürden in ihrem Lernprozess dar. Für die Aktivität des Beweisverstehens gibt es zwar Operationalisierungsmodelle, um das Ergebnis des Verstehensprozesses zu messen, jedoch nur wenige Studien, die Einflussfaktoren auf den Verstehensprozess identifizieren. An diesem Punkt setzt diese Studie an und untersucht zu einem Beweis im Gebiet der Analysis, welche individuellen Faktoren das Beweisverständnis prädizieren, welche Varianten der Strategie „Illustrieren am Beispiel“ das Beweisverstehen unterstützen und wie Studierende Beispiele in Verstehensprozessen nutzen. Diese Ziele werden in den folgenden Fragen konkretisiert:

1. Inwiefern kann das Beweisverständnis im Gebiet der Analysis durch inhaltliches Vorwissen und Vorleistungen in Form der Gesamt-Abiturnote prädiziert werden?

Wir erwarten, dass das inhaltliche Vorwissen das Beweisverständnis stark prädiziert (vgl. Ergebnisse zum Validieren von Sommerhoff 2017). Da die Vorleistungen in Form der Gesamt-Abiturnote einen guten Indikator für die Leistungsfähigkeit einer Person darstellen (vgl. Trapmann et al. 2007), erwarten wir, dass die Gesamt-Abiturnote einen Teil der Varianz im Beweisverständnis zwischen Studierenden aufklärt (vgl. Neuhaus und Rach 2019).

Wie im Forschungsstand dargestellt, wird das Illustrieren am Beispiel als eine Strategie von Expertinnen und Experten beim Beweisverstehen angewendet. Darüber hinaus ist das Illustrieren am Beispiel ein viel praktiziertes und empfohlenes Vorgehen bei Mathematikdozierenden. Obwohl die grundlegende Annahme, dass das Illustrieren am Beispiel eine sinnvolle Aktivität zur Unterstützung des Beweisverstehens durch Lernende ist, nicht zwingend belegt ist, liegt sie der gängigen Lehrpraxis zugrunde und ist auch unter Rückgriff auf vorliegende Analysen von Beweisverstehensprozessen argumentativ stützbar. Wir fragen daher, in welcher Form eine entsprechende Aktivität im Verstehensprozess besonders wirksam werden kann:

2. In welcher Darstellungsform und mit welcher Lernaktivität verknüpft unterstützen zeilenweise illustrierende Beispiele das Beweisverständnis im Gebiet der Analysis?

Aufgrund der Ergebnisse von Ostsieker (2020) erwarten wir, dass eine mögliche graphische Darstellung das Beweisverständnis stärker unterstützt als eine symbolische Darstellung des Beispiels. Nach den Ergebnissen von Chi und Wylie (2014) und Ufer (2015) gehen wir weiter davon aus, dass eine konstruktive Lernaktivität zu einer stärkeren Auseinandersetzung mit dem Beweis führt und 
damit zu einem besseren Beweisverständnis als eine passive Lernaktivität. Eine Interaktion aus Darstellungsform und Lernaktivität erwarten wir nicht.

Die Arbeit von Ahmadpour et al. (2019) ist ein konkretes Beispiel dafür, dass der Blick auf die gesamten Verstehensprozesse ein vielschichtigeres Bild von Beweisverstehen entwirft als der alleinige Blick auf mögliche Endzustände des Prozesses. Dies motiviert einen im Rahmen der vorgestellten Studie eher explorativen, qualitativen Blick auf die einzelnen Bearbeitungsprozesse unter der Fragestellung:

3. Inwieweit geben die Bearbeitungsprozesse weiteren Aufschluss über spezifische Bedingungen für die Wirksamkeit der Strategie „Illustrieren am Beispiel“ für das Beweisverstehen?

\section{Methodisches Vorgehen}

\subsection{Stichprobe}

Die Stichprobe bestand aus 166 Studierenden von zwei deutschen Universitäten. Aus Universität 1 haben 66 Studierende teilgenommen, aus Universität 2 haben 100 Studierende teilgenommen. Die Studierenden waren in den folgenden Studiengängen eingeschrieben: Bachelor Mathematik $(n=20)$, Bachelor Wirtschaftsmathematik ( $n=21)$, gymnasiales Lehramt $(n=57)$, Regionalschullehramt $(n=16)$, Bachelor Physik $(n=48)$ und andere Studiengänge $(n=4)$. Jeweils 41,6\% der Studierenden waren im ersten und zweiten Studienjahr eingeschrieben, die anderen 16,9\% (gerundet) waren in höheren Studienjahren eingeschrieben.

\subsection{Erfassung individueller Merkmale}

Um individuelle Merkmale zu identifizieren, die das Beweisverständnis prädizieren, wurde die Vorleistung der Studierenden anhand ihrer Gesamt-Abiturnote und deren mathematisches Vorwissen anhand eines Tests erfasst. Die Gesamt-Abiturnote reicht von 1,0 (sehr gut) bis 4,0 (ausreichend) $(M=1,95 ; S D=0,56)$ und ist ein guter Indikator für die schulischen Vorleistungen, da es sich im Gegensatz zu einzelnen Fachnoten um ein aggregiertes Maß handelt und als solches weniger anfällig für Messfehler ist (Trapmann et al. 2007). Der Vorwissenstest hat das Ziel, das inhaltliche Vorwissen im Bereich reellwertiger Folgen zu messen. Der Schwerpunkt liegt insbesondere auf dem konzeptuellen Wissen, z. B. der Identifikation von konvergenten und divergenten Folgen (siehe auch Rach 2014), und weniger auf dem prozeduralen Wissen. Der Vorwissenstest besteht aus sechs multiple-choice und zwei offenen Items, deren Bearbeitung jeweils dichotom kodiert wurde, also es wurden jeweils 0 Punkte oder 1 Punkt vergeben. Die offenen Items wurden anhand eines Kodierschemas bewertet. Ein Beispielitem ist in Abb. 2 dargestellt.

Aufgrund von Zeitproblemen bei der Erhebung wurde der Vorwissenstest nur von 99 Studierenden (66 Studierenden der Universität 1; 33 der Universität 2) bearbeitet. Der Vorwissenstest mit acht Items war im Schwierigkeitsgrad angemessen für die 
Vervollständigen Sie die folgende Definition:

Sei $\left(a_{n}\right)_{n \in \mathbb{N}}$ eine Folge in $\mathbb{R}$. Die Folge $\left(a_{n}\right)_{n \in \mathbb{N}}$ ist monoton fallend, wenn $\ldots$

Abb. 2 Beispielitem zur Erfassung des Vorwissens

Studierenden: $M=5,15 ; S D=2,26$; Cronbachs $\alpha=0,75$. Die Gesamt-Abiturnote und das Vorwissen korrelieren mit mittlerer Stärke miteinander $(r=-0,36$; $p<$ $0,001 ; n=91)$.

\subsection{Erfassung von Beweisverständnis}

Für den Bereich der Hochschulmathematik haben Mejía-Ramos et al. (2012) ein Operationalisierungsmodell von Beweisverständnis entwickelt. Dieses Modell ist ein in der Literatur verbreitetes Operationalisierungsmodell für Beweisverständnis und wird daher als Basis für die Erhebung von Beweisverständnis der hier vorgestellten Studie verwendet.

Mejía-Ramos et al. (2012) teilen Beweisverständnis in zwei Ebenen ein, die wiederum in verschiedene Facetten unterteilt sind. In der lokalen Ebene werden nur einzelne bzw. wenige Zeilen eines Beweises betrachtet, während in der ganzheitlichen Ebene der Beweis als Gesamtkonstrukt erfasst wird. Das Modell von MejíaRamos et al. (2012) bildet auf lokaler und auf ganzheitlicher Ebene sowohl Aspekte eines Verstehens-von-Beweisen als auch eines Verstehens-durch-Beweise ab. Die von uns betrachtete Strategie ,Illustrieren an Beispielen“ wird bei Mejía-Ramos et al. (2012) als Indikator für das Vorliegen von Beweisverständnis verwendet. Damit die Gruppe, die nicht zur Beweisillustration aufgefordert war, keinen expliziten Nachteil im Test hat, wurden in unserer Studie keine Items für den Beweisverständnistest verwendet, die sich diesem Indikator zuordnen lassen.

Satz. Jede Folge $\left(a_{n}\right)_{n \in \mathbb{N}}$ in $\mathbb{R}$ hat eine monotone Teilfolge.

Beweis.

(1) Wir nennen einen Index $n \in \mathbb{N}$ eine Gipfelstelle der Folge $\left(a_{n}\right)$, falls für alle $m>n$ gilt $a_{m}<a_{n}$.

(2) Falls die Folge $\left(a_{n}\right)$ unendlich viele Gipfelstellen hat, dann definieren diese eine monoton fallende Teilfolge.

(3) Sei nun angenommen, dass $\left(a_{n}\right)$ nur endlich viele Gipfelstellen hat.

(4) Wähle den kleinsten Index $k_{1}$, der nach den endlich vielen Gipfelstellen kommt.

(5) Dann ist $k_{1}$ keine Gipfelstelle.

(6) Daher existiert ein $k_{2}>k_{1}$ mit $a_{k_{2}} \geq a_{k_{1}}$. Sei $k_{2}$ die kleinste solche Stelle.

(7) Da auch $k_{2}$ keine Gipfelstelle ist, existiert ein $k_{3}>k_{2}$ mit $a_{k_{3}} \geq a_{k_{2}}$. Sei $k_{3}$ die kleinste solche Stelle.

(8) Fährt man induktiv auf diese Weise fort, so entsteht eine monoton steigende Teilfolge von $\left(a_{n}\right)$.

Abb. 3 Satz und Beweis zu monotonen Teilfolgen. Die Beweisschritte wurden nummeriert, um in der Intervention auf sie Bezug nehmen zu können 
Für die Studie wurde ein Beweis verwendet, der in der Struktur und in den gewählten Mitteln typisch für den Stil eines Beweises im Rahmen einer Analysisvorlesung ist. Konkret handelt es sich um den folgenden Satz über monotone Teilfolgen: Jede Folge $\left(a_{n}\right)_{n \in \mathbb{N}}$ in $\mathbb{R}$ hat eine monotone Teilfolge. Diese Aussage lässt sich als Hilfsmittel nutzen, um den Satz von Bolzano-Weierstraß zu beweisen (für einen solchen Zugang siehe z.B. Heuser 1980, S. 156). In den Lerngruppen, in denen diese Studie durchgeführt wurde, war der Satz von Bolzano-Weierstraß allerdings auf andere Weise behandelt worden.

Wir erläutern den Gang des Beweises (siehe Abb. 3), um aufzuzeigen, dass er eine Reihe von typischen Strukturelementen von Beweisen besitzt:

- Der Beweis führt zunächst einen neuen Begriff ein („Gipfelstelle“). Dieser wird nur lokal innerhalb des Beweises benötigt, spielt in der nachfolgenden Argumentation aber eine Schlüsselrolle. Für das Beweisverständnis ist hier also auch das Verstehen einer Definition erforderlich sowie im weiteren Verlauf das Begriffsverständnis zu diesem neuen Begriff.

- Sodann nimmt der Beweis eine Fallunterscheidung vor, je nachdem, ob die gegebene Folge unendlich viele oder endlich viele Gipfelstellen hat. Die beiden Fälle sind hier nicht Varianten voneinander, sondern werden auf substantiell verschiedene Weise bearbeitet. Für das Verstehen des Beweises ist es erforderlich, den so gegebenen logischen Rahmen zu verstehen.

- Falls die Folge unendlich viele Gipfelstellen hat, so liefern diese eine (sogar streng) monoton fallende Teilfolge und der Beweis ist bereits erbracht. Um dies zu verstehen, ist Begriffsverständnis (zu Gipfelstellen) erforderlich.

- Der Fall endlich vieler Gipfelstellen macht den Hauptteil des Beweises aus. Durch ein iteratives Vorgehen werden Folgenglieder ausgewählt, die nach den endlich vielen Gipfelstellen liegen, und aus ihnen wird eine monoton steigende Teilfolge konstruiert. Die definierende Eigenschaft von Gipfelstellen sichert dabei, dass die Konstruktion nach jedem gewählten Folgenglied induktiv weitergeführt werden kann.

Der oben beschriebene Beweis ist genügend reichhaltig, um eine Reihe von Facetten des Beweisverständnisses aus dem Modell von Mejía-Ramos et al. (2012) testen zu können. Zusätzlich wurden zwei über das Modell hinausgehende Facetten (zum Tiefenverständnis der Konstruktion und zu Randfällen) verwendet.

Lokales Beweisverständnis:

1. Logical status of statements and proof framework - Identify the proof framework: Es wurde erfragt, ob der Beweis eine Fallunterscheidung oder ein Widerspruchsargument enthält.

2. Logical status of statements and proofframework - Identify purpose of a sentence within a proof framework: Hier war zu entscheiden, ob in Schritt (1) eine Behauptung formuliert, eine Aussage bewiesen, ein Begriff definiert oder eine Voraussetzung wiederholt wurde.

3. Justification of claims - Make explicit an implicit warrant in the proof: Im Beweis wird in Schritt (2) gesagt: „Falls die Folge $\left(a_{n}\right)$ unendlich viele Gipfelstellen hat, 
Könnte man den Beweis auf die prinzipiell selbe Weise führen, wenn man statt Gipfelstellen einen analog definierten Begriff Talstellen verwenden würde?

Ja, wenn man im Beweis »fallend « und »steigend « austauscht und an bestimmten Stellen die Ungleichheitszeichen umkehrt.

Ja, wenn man im Beweis »fallend « und »steigend « austauscht und an bestimmten Stellen die Reihenfolge von Schritten ändert.

Nein, weil der Beweis dann nur funktioniert, wenn die gegebene Folge eine monoton fallende Teilfolge besitzt.

Nein, weil der Beweis dann nur funktioniert, wenn die gegebene Folge eine monoton steigende Teilfolge besitzt.

Abb. 4 Beispielitem aus dem Beweisverständnistest zu weiteren Aspekten (8)

dann definieren diese eine monoton fallende Teilfolge." Der Garant, der in der definierenden Eigenschaft von Gipfelstellen liegt, war hier zu explizieren.

4. Meaning of terms and statements - Identify trivial implications of a given statement: In Schritt (4) des Beweises wird ein Index $k_{1}$ gewählt. Es war zu entscheiden, welche von vier gegebenen Aussagen über Indizes $k>k_{1}$ sich daraus folgern lässt.

5. Justification of claims - Identify the specific data supporting the given claim: Es musste angegeben werden, aus welchen Angaben im Beweis die Existenz des benötigten Index und damit Folgenglieds folgt (siehe Abb. 7, Anhang A).

Ganzheitliches Beweisverständnis:

6. Ask students to partition a proof into modules: Der Beweis war in drei inhaltlich zusammenhängende Teilabschnitte zu zerlegen, die mit inhaltlich passenden Überschriften zu versehen waren (siehe Abb. 8, Anhang A).

7. Summarizing via high-level ideas - Identify a good summary of a key sub-proof in the proof: Die Studierenden hatten zu entscheiden, welche angegebene Aussage der Beweis zusätzlich zum explizit formulierten Satz noch zeigt.

Weitere Aspekte:

8. Tiefenverständnis der Konstruktion: Um zu erfassen, ob die Studierenden die Rolle der Gipfelstellen für die durch den Beweis gegebene Konstruktion einer monotonen Teilfolge tiefergehend verstanden haben, wurde gefragt, ob (und mit welchen Änderungen) man den Beweis mit einem analog definierten Begriff ,,Talstelle" führen könnte (siehe Abb. 4).

9. Verständnis von Grenzfällen: Um auszuloten, ob die Studierenden den Beweis bis hin zu Randfällen verstanden haben, wurde gefragt, was der Beweis leistet, wenn die gegebene Folge konstant ist.

Acht der neun Items sind im single-multiple-choice Format gestellt. Diese acht Items wurden dichotom codiert, d.h. 0 Punkte oder 1 Punkt wurden vergeben. Die Anforderung beim anderen Item war es, den Beweis in drei zusammenhängende Abschnitte einzuteilen und diese Abschnitte mit einer Überschrift zu versehen. Dieses Item wurde von den Autorinnen und Autoren doppelt kodiert und eine gute Übereinstimmungsrate von größer als $80 \%$ festgestellt; konsensuell wurde dann ein Wert 
festgelegt. Bei diesem Item „Einteilung des Beweises“ (siehe Abb. 8, Anhang A) wurden neben 0 Punkten und 1 Punkt auch 0,5 Punkte vergeben, wenn die Einteilung des Beweises korrekt war, aber nicht alle Überschriften den Kern der Beweisteile trafen.

Cronbachs $\alpha$ für den Beweisverständnistest beträgt 0,68 (bei $N=166$ ). Aufgrund der Breite des Konstruktes ist dieser relativ niedrige Wert nicht verwunderlich. Da die Trennschärfen für jedes der neun Items über 0,22 liegen und eine explorative Faktorenanalyse keinen Hinweis auf eine mehrdimensionale Struktur gibt, bilden wir eine Gesamtskala für das Konstrukt Beweisverständnis. Die deskriptive Analyse zeigt auf, dass der Beweisverständnistest im Mittel angemessen für die Stichprobe war: $M=4,40 ; S D=2,01$; die Lösungshäufigkeiten der Items liegen zwischen $15 \%$ und $80 \%$.

\subsection{Design}

Der Erstautor und die Zweitautorin haben die Untersuchung nach einem festgelegten Ablauf mit Zeitvorgaben und Anweisungen durchgeführt. Für die Implementation der unterschiedlichen Varianten wurden insgesamt fünf Manuale verwendet (vgl. Abb. 5). Die Manuale sind folgendermaßen aufgebaut: Es wurde der Beweis präsentiert, dann wurden die Studierenden in allen Varianten aufgefordert, den Beweis anhand der vorgegebenen Intervention durchzuarbeiten (13 min) und danach innerhalb von $15 \mathrm{~min}$ den Verständnistest für den Beweis zu bearbeiten. An der ersten Universität haben die Studierenden zu Beginn den Vorwissenstest innerhalb von zwölf Minuten bearbeitet. Aufgrund der Tatsache, dass bei der Durchführung an Universität 2 weniger Zeit als an Universität 1 zur Verfügung stand, haben wir aus pragmatischen Gründen den Vorwissenstest an das Ende des Manuals gestellt. Aus Universität 2 haben nur noch wenige Studierende am Vorwissenstest teilgenommen und weniger Daten aus dieser Durchführung im Vergleich zur Universität 1 sind in die Beantwortung von Forschungsfrage 1 eingeflossen.

Die beiden Interventionsbedingungen (graphisch versus symbolisch und konstruktiv versus passiv) wurden in vier der Manuale umgesetzt. Dazu wurde im Fall 1 des Beweises in allen Bedingungen ein Beispiel präsentiert, das sich je nach Bedingung nur in der Darstellungsform unterschied. Diese Maßnahme sollte Studierenden deutlich machen, wie ein korrektes und hilfreiches Beispiel zur Illustration von Beweiszeilen genutzt werden kann (vgl. Iannone et al. 2011). Dann wurden die

Abb. 5 Ablauf der Implementation

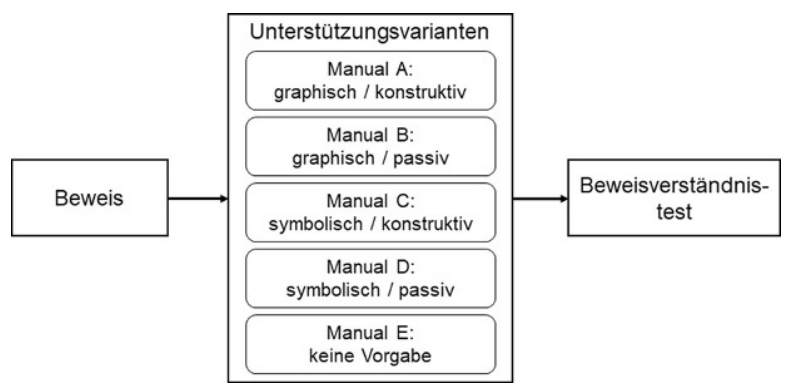


Lesen Sie sich den folgenden Satz mit Beweis und die Beispiele gründlich durch.

Satz: Jede Folge $\left(a_{n}\right)_{n \in \mathbb{N}}$ in $\mathbb{R}$ hat eine monotone Teilfolge.

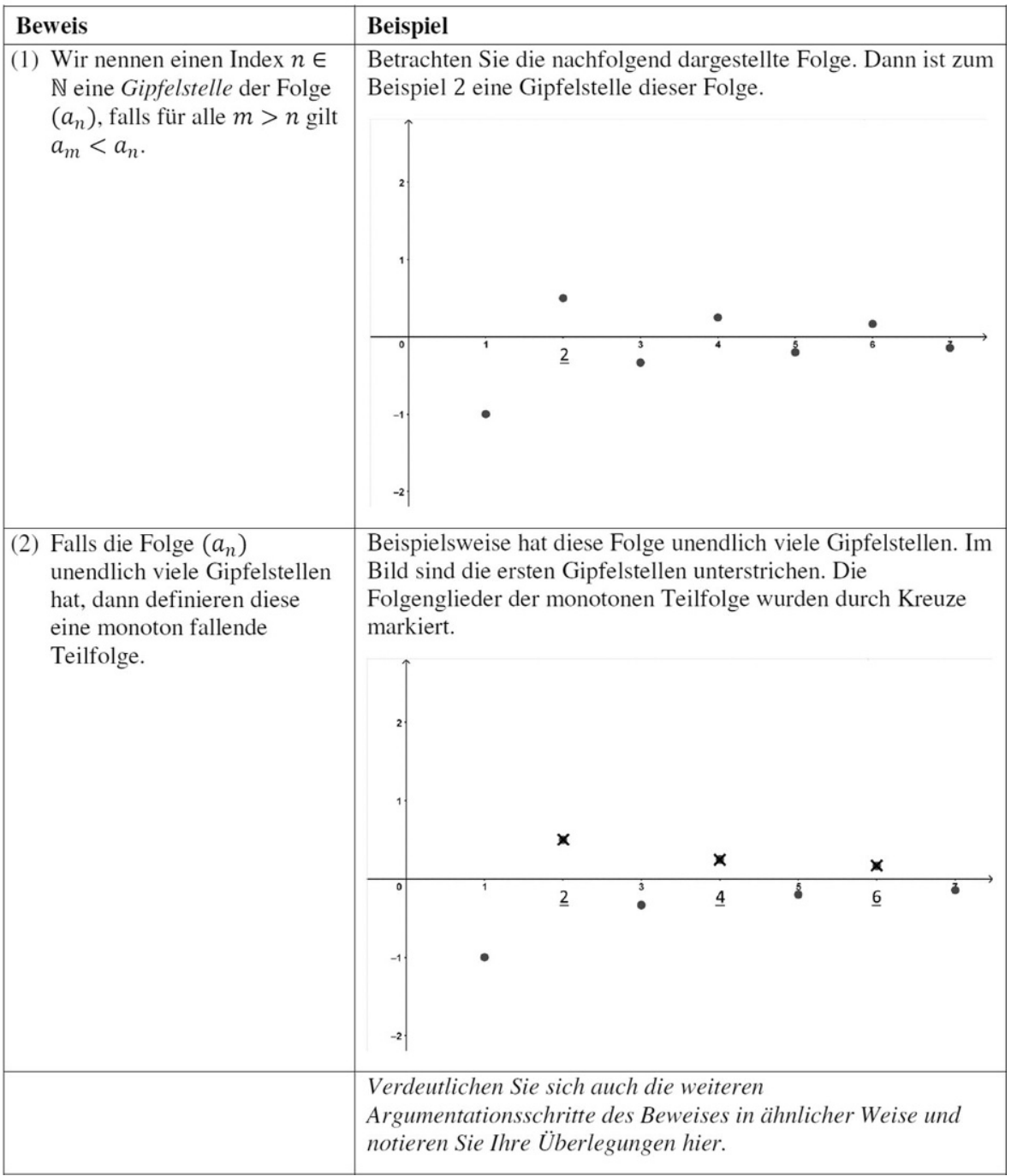

Abb. 6 Ausschnitt aus dem Manual zur Bedingung ,konstruktiv/graphisch“, Fall 1 des Beweises

Studierenden der Bedingung „,konstruktiv“ aufgefordert, ein Beispiel für Fall 2 zu konstruieren, während in der Bedingung ,passiv“ dieses Beispiel schon vorgegeben war. Ein Ausschnitt zum Manual graphisch/konstruktiv ist in Abb. 6 dargestellt. Die Beispielfolge ist dort als Graph gegeben, in dem eine Gipfelstelle markiert ist. In der Bedingung ,symbolisch“ war die Beispielfolge stattdessen durch die Gleichung $a_{n}=(-1)^{n} \cdot \frac{1}{n}$ dargestellt und es war 2 als Gipfelstelle angegeben. Die nächsten 
Tab. 1 Anzahl der Studierenden pro Bedingung, ohne Manual E

\begin{tabular}{lll}
\hline & Konstruktiv & Passiv \\
\hline Graphisch & Manual A: 40 & Manual B: 35 \\
Symbolisch & Manual C: 35 & Manual D: 32 \\
\hline
\end{tabular}

Seiten des Manuals waren wiederum in zwei Spalten geteilt, wobei die erste Spalte die einzelnen Beweisschritte enthielt, während die zweite Spalte in der konstruktiven Bedingung leer war und in der passiven Bedingung die Illustration der Schritte wie in Fall 1 erfolgte. Das fünfte eingesetzte Manual war offener gestaltet, d.h. auch für Fall 1 war kein Beispiel vorgegeben. Die Studierenden, die dieses Manual bearbeitet haben, wurden aufgefordert, den Beweis zu lesen, zu verstehen und Beispiele zu bilden.

Die Studierenden wurden randomisiert und möglichst gleichmäßig auf die fünf Manuale verteilt (siehe Tab. 1): Die Ergebnisse der Bedingung ohne Vorgabe (Manual E) werden nur für die Prädiktion von Beweisverständnis durch individuelle Merkmale verwendet (Forschungsfrage 1). Die randomisierte Verteilung der Studierenden auf die beiden Bedingungen (1) Darstellungsform und (2) Lernaktivität hat nur z.T. dazu geführt, dass sich die Gruppen in den Kontrollvariablen nicht substanziell unterscheiden: Während keine signifikanten Unterschiede bezüglich der Kontrollvariable „Vorleistungen“, gemessen durch die Gesamt-Abiturnote, festzustellen ist (jeweils $p>0,20 ; \eta^{2}<0,015$ für beide Bedingungen), unterscheiden sich die beiden Bedingungen bezüglich des „Vorwissens“ (Darstellungsform: $F(1,94)=6,09$; $p<0,05 ; \eta^{2}=0,061$; Lernaktivität: $\left.F(1,94)=2,79 ; p=0,10 ; \eta^{2}=0,029\right)$. Um die Effekte der Bedingungen später zu überprüfen, haben wir die Vorleistungen und das Vorwissen kontrolliert. Nur drei Studierende der offenen Gruppe (Manual E, $n=24$, nur an einer Hochschule eingesetzt) haben den Vorwissenstest bearbeitet, so dass bei dieser Gruppe keine Aussagen über deren Vorwissen möglich sind.

In der konstruktiven Bedingung waren die Studierenden dazu angehalten, ein eigenständiges Beispiel, in diesem Fall eine reellwertige Folge, zu konstruieren, das unter Fall 2 des Beweises fällt. Anhand dieses Beispiels sollten die Studierenden dann den Beweis nachvollziehen. Da sie Beweiszeile für Beweiszeile am Beispiel konkretisieren sollten, ermöglichen die Bearbeitungen aus dieser Gruppe auch einen genaueren Einblick in den Bearbeitungs- und Verstehensprozess, was mit Blick auf Forschungsfrage 3 von Interesse ist. In der passiven Bedingung ist dieses Beispiel schon gegeben und die Studierenden werden nur dazu aufgefordert, das Beispiel zu lesen. Die Bedingung graphisch versus symbolisch unterschied sich darin, in welcher Darstellungsform das Beispiel gegeben war bzw. konstruiert werden sollte.

Als Interventionskontrolle wurde überprüft, in welcher Darstellungsform die Studierenden die Beispiele konstruiert haben. Insgesamt 10 Studierende haben keine Aufzeichnungen angefertigt, die kodiert werden konnten, und sind somit auch aus den Analysen für Forschungsfrage 3 herausgefallen. In der graphischen Gruppe (nun $n=36$; Manual A) nutzten zwei Studierende ein symbolisch dargestelltes Beispiel und vier Studierende konstruierten kein explizites Beispiel, sondern nutzen nur Selbsterklärungen (vgl. Chi et al. 1989; Rittle-Johnson et al. 2017). In der symbolischen Gruppe (nun $n=29$; Manual C) nutzten drei Personen auch ansatzweise 
graphische Darstellungen, diese waren aber entweder nur Visualisierungen zur Verdeutlichung $(n=2)$ oder keine konkreten Beispiele $(n=1)$. Außerdem nutzen sechs Personen nur Selbsterklärungen und bei drei Personen konnten nur unverständliche Ansätze identifiziert werden. Insgesamt haben sich die meisten Studierenden aber an die ihnen zugewiesene Bedingung gehalten.

\subsection{Datenanalyse zur Prädiktion von Beweisverständnis und zum Effekt der Unterstützungsvarianten}

Um die Forschungsfragen 1 und 2 zu beantworten, wird die Software R verwendet. Da einige fehlende Werte vor allem beim Vorwissen vorliegen, werden diese durch den FIML (Full Information Likelihood)-Algorithmus im Package lavaan geschätzt (Rosseel 2012). Zur Schätzung der fehlenden Daten wird ein Hintergrundmodell verwendet, das für alle Auswertungen jeweils alle verwendeten Variablen (für Forschungsfrage 1: Vorwissen, Gesamt-Abiturnote, Beweisverständnis; für Forschungsfrage 2: Vorwissen, Gesamt-Abiturnote, Beweisverständnis, Darstellungsform, Lernaktivität) enthält.

Um Unterschiede im Beweisverständnis durch individuelle Merkmale (Forschungsfrage 1) vorherzusagen, werden zwei lineare Regressionsmodelle gerechnet, wobei jeweils das Beweisverständnis die abhängige Variable bildet. Im Modell 1 werden nur die Vorleistungen in Form der Gesamt-Abiturnote und im Modell 2 das mathematische Vorwissen und die Vorleistungen in Form der Gesamt-Abiturnote als unabhängige Variable verwendet. Da die Gesamt-Abiturnote und das Vorwissen nur mit mittlerer Stärke miteinander korrelieren $(r=-0,36 ; p<0,001)$, ist die Gefahr, dass die Voraussetzungen für Regressionsanalysen aufgrund von Multikollinearität verletzt sind, als eher gering einzuschätzen.

Die Effekte der Intervention (Forschungsfrage 2) werden ebenfalls in einem linearen Regressionsmodell mit Beweisverständnis als abhängige Variable und den beiden Faktoren Darstellungsform (mit den Stufen ,graphisch“ und ,symbolisch“) und Lernaktivität (mit den Stufen „,konstruktiv“ und ,passiv“) analysiert. Im Hintergrundmodell wird neben den schon erwähnten Variablen (Vorwissen, GesamtAbiturnote, Beweisverständnis, Darstellungsform, Lernaktivität) ebenfalls eine Interaktion aus Darstellungsform und Lernaktivität mit aufgenommen, obwohl hierzu keine Hypothese gebildet wurde. Dieses ist notwendig, um Modelle mit und ohne Interaktion vergleichen zu können. Dass Interaktionen zwischen den beiden Faktoren eine wichtige Rolle spielen könnten, wird bei der Betrachtung der deskriptiven Werte für das Beweisverständnis (Abschn. 5.2) und der Analyse der Beweisverstehensprozesse (Abschn. 5.3) deutlich.

\subsection{Kodierung des Bearbeitungsprozesses}

Um einen Einblick in den konkreten Bearbeitungsprozess zu erhalten, wurden die von den Studierenden der konstruktiven Bedingung selbst konstruierten Beispiele (für den zweiten Fall, den Hauptfall des Beweises) unter zwei Aspekten kategorisiert: Ein Beispiel wird als ,hilfreich“ eingestuft, wenn es den Prozess des Beweisverstehens unterstützt. Dies bedeutet hier, dass das Beispiel konkret die Bedeutung jeder 
einzelnen Beweiszeile, insbesondere der darin verwendeten allgemeinen Prozeduren, verdeutlicht und die Anwendung jeder einzelnen Beweiszeile auf das Beispiel nicht trivial ist (eine konstante Folge würde daher als wenig hilfreich eingestuft). „Korrekt" ist ein Beispiel, wenn es in den einzelnen Beweisschritten im Wesentlichen richtig angewendet wurde.

Somit muss das erste Merkmal durch Expertinnen und Experten zum Beweisverstehen eingeschätzt werden, während das zweite Merkmal rein fachmathematisch zu sehen ist. Wir beschreiben nun die Kodierung dieser beiden Aspekte im Einzelnen:

Aspekt 1 Wie hilfreich ist das Beispiel zum Verständnis des Beweises aus Expertensicht? Hierzu wurden zunächst deduktiv vier Klassen gebildet, welche den erwarteten Konstruktionen entsprechen und in absteigender Reihenfolge vier Grade der Eigenschaft ,hilfreich“ entsprechen ( $3=$ sehr hilfreich, $2=$ hilfreich, $1=$ wenig hilfreich, $0=$ nicht hilfreich):

- Code 3: Folgen, bei denen das im Beweis gezeigte Vorgehen eine echte Teilfolge auswählt, die zudem nicht konstant ist. Dies ist etwa bei alternierend wachsenden Folgen wie $(-1)^{n} n+$ const der Fall und auch bei Folgen, die sich schließlich (d.h. ab einem bestimmten Index) so verhalten. Expertinnen und Experten würden ein Beispiel dieser Art wählen, um den Auswahlprozess sichtbar zu machen. Wir betrachten sie daher als sehr hilfreich.

- Code 2: Folgen, bei denen das im Beweis gezeigte Vorgehen eine echte Teilfolge auswählt, die aber konstant ist. Dies ist etwa bei $(-1)^{n}+$ const der Fall (sowie bei Folgen, die sich schließlich so verhalten). Wir betrachten solche Beispiele als hilfreich, aber nicht im selben Maße wie in der vorigen Kategorie, da die Auswahl einer konstanten Folge einen Spezialfall darstellt, der möglicherweise eine zu enge Sicht auf den Beweis vermittelt.

- Code 1: Folgen, die von vorneherein monoton steigend (oder sogar konstant) sind. Da hier der Auswahlprozess zu einfach ist (die Folge ist gleich der Teilfolge), betrachten wir sie als wenig hilfreich.

- Code 0: Folgen, bei denen keine Bildungsvorschrift zu erkennen ist und die auch keine begleitenden Erläuterungen haben. Dies betrachten wir als nicht hilfreich. Ebenfalls in dieser Kategorie liegen Folgen, die nicht zum betrachteten Fall passen (weil sie unendlich viele Gipfelstellen haben).

Induktiv wurde die Kategorisierung so erweitert, dass auch die weiteren in unserer Stichprobe vorkommenden Bearbeitungen erfasst werden konnten:

- zu Code 1: Es wurde kein Beispiel konstruiert, sondern Selbsterklärungsansätze auf abstrakter Ebene angegeben. Diese Ansätze sind mindestens teilweise korrekt und insofern potenziell hilfreich.

- zи Code 0: Es wurde kein Beispiel konstruiert, sondern Selbsterklärungsansätze auf abstrakter Ebene angegeben, die allerdings inkorrekt sind. Diese sind nicht hilfreich.

Aspekt 2 Wie korrekt wurde mit dem angegebenen Beispiel umgegangen? Hierfür sind drei Codes ( $1=$ korrekt, $0,5=$ teilweise korrekt, $0=$ inkorrekt) vergeben worden: 
- Code 1: Der Beweis wird an dem gewählten Beispiel im Wesentlichen korrekt durchgeführt, d.h. im Beispiel wird die Teilfolge nach dem im Beweis gegebenen Verfahren ausgewählt. Kleinere Fehler, die die Konstruktion als Ganzes nicht beeinträchtigen, bleiben dabei unberücksichtigt.

- Code 0,5: Der Beweis wird an dem gewählten Beispiel nur teilweise durchgeführt. Dieser Code wird z. B. dann vergeben, wenn der Index $k_{3}$ nicht als der kleinste solche Index gewählt wird oder wenn das Beispiel inkonsistent verwendet wird (z.B. Folgenglieder verschwinden in späteren Schritten).

- Code 0: Der Beweis wird an dem gewählten Beispiel nur in Ansätzen verfolgt, die aber unbrauchbar sind. Dieser Code wird auch dann vergeben, wenn kein Beispiel oder ein unpassendes Beispiel angegeben wurde.

Der Code ist auf die individuelle Bearbeitung bezogen - so kann etwa ein an sich sehr hilfreiches Beispiel vom alternierend-wachsenden Typ (Code 3 im Aspekt „hilfreich“) mehr oder weniger korrekt bearbeitet worden sein. Bearbeitungen, die unter dem Aspekt ,hilfreich“ Code 0 erhalten, erhalten unter dem Aspekt ,korrekt" automatisch ebenfalls Code 0. Das in den vier Manualen, in denen die beiden Interventionsbedingungen umgesetzt wurden, vorgegebene Beispiel für Fall 1 des Beweises (siehe Abb. 6) ist gemäß dieser Kodierung ein sehr hilfreiches und korrektes Beispiel.

Dieses ausführliche Kodierschema wurde durch den Erstautor und die Zweitautorin dieses Beitrages entwickelt und angewendet und dann zusätzlich von der Drittautorin verwendet. Diese Doppelkodierung weist eine gute Übereinstimmungsrate von $87 \%$ (Beispiel hilfreich) und $82 \%$ (Beispiel korrekt) auf. Nach einem Konsensverfahren wurden die endgültigen Kodierungen festgelegt.

\section{Ergebnisse}

\subsection{Prädiktion von Beweisverständnis}

In Modell 1 (Gesamt-Abiturnote als Prädiktor) können 9,6\% der Varianz im gezeigten Beweisverständnis erklärt werden: Gesamt-Abiturnote: $\beta=-0,31 ; p<0,001$. Studierende, die mit schlechteren schulischen Vorleistungen ihr Studium starten, zeigen somit im Mittel ein geringeres Beweisverständnis. In Modell 2 (GesamtAbiturnote und Vorwissen als Prädiktoren) können 37,6\% der Varianz durch die individuellen Merkmale erklärt werden: Gesamt-Abiturnote: $\beta=-0,12 ; p<0,001$; Vorwissen: $\beta=0,56 ; p<0,01$.

Wie erwartet, kann insgesamt ein substanzieller Anteil an Varianz im Beweisverständnis durch individuelle Merkmale aufgeklärt werden. Eine niedrige GesamtAbiturnote, d.h. gute schulische Leistungen, und ein hohes inhaltliches Vorwissen gehen mit einem hohen Beweisverständnis einher. 
Tab. 2 Beweisverständnis ( $M$ und $S D$, Maximum 9 Punkte), aufgetragen nach den beiden Faktoren

\begin{tabular}{lll}
\hline & Graphisch & Symbolisch \\
\hline Konstruktiv & $4,51(2,13)$ & $4,19(1,95)$ \\
Passiv & $5,24(2,11)$ & $4,08(1,98)$ \\
\hline
\end{tabular}

Die Unterschiede im Beweisverständnis zwischen den Gruppen sind nicht signifikant

\subsection{Effekte der Unterstützungsmaßnahmen}

Als signifikanter Faktor zur Erklärung von Unterschieden im Beweisverständnis kann nur das Vorwissen identifiziert werden. Entgegen den Erwartungen können keine Haupteffekte durch die beiden Faktoren (graphisch versus symbolisch $p=0,99$; konstruktiv versus passiv $p=0,88$ ) berichtet werden. Anhand der deskriptiven Ergebnisse, dargestellt in Tab. 2, könnte eine Interaktion aus Darstellungsform und Lernaktivität vermutet werden und zwar, dass bei der graphischen Darstellungsform die passive Lernaktivität der konstruktiven Lernaktivität überlegen ist. Die beiden Modelle mit und ohne Interaktion unterscheiden sich bei dieser Studie jedoch nicht signifikant voneinander $\left(\chi^{2}(1)=2,51 ; p=0,11\right)$. Diese unerwartete, explorative Vermutung muss in weiteren Studien überprüft werden und lässt sich durch die hier präsentierten Daten weder belegen noch verwerfen.

Die Ergebnisse geben keine Hinweise, welche Varianten lernförderlicher sind, um anhand des Illustrierens am Beispiel das Beweisverstehen zu unterstützen. Diese Ergebnisse sind möglicherweise darauf zurückzuführen, dass die Studierenden zu wenig Vorwissen besitzen, um beispielsweise in der Bedingung ,graphisch/konstruktiv“ ein geeignetes Beispiel zu entwickeln und für ihren Beweisverstehensprozess nutzbar zu machen. Um diese mögliche Begründung weiter auszuführen, betrachten wir den konkreten Bearbeitungsprozess der Studierenden.

\subsection{Konkreter Bearbeitungsprozess}

Diese Analysen haben einen explorativen Charakter. Anhand des Forschungsstandes hatten wir erwartet, dass die Bedingung ,graphisch/konstruktiv“ das Beweisverstehen am besten unterstützt. Die in Abschn. 5.2 dargestellten Ergebnisse stützen diese Annahme jedoch nicht. Aus diesem Grund sind die konkreten Bearbeitungsprozesse der Studierenden von besonderem Interesse. Unsere Analyse konkretisiert sich in der Frage, inwiefern die Studierenden die Unterstützungsmaßnahme sinnvoll nutzen konnten. Zur Analyse des Bearbeitungsprozesses haben wir nach dem in Abschn. 4.6 beschriebenen Verfahren alle Beispiele (bzw. Selbsterklärungen), die in den beiden „konstruktiven“ Gruppen erstellt wurden, nach den beiden Kriterien „hilfreich“ und „korrekt" kodiert.

Tab. 3 und 4 zeigen die Ergebnisse der Kodierung. Im Anhang B finden sich Ausschnitte aus drei Studierendenbearbeitungen und deren Kodierung (Bearbeitung 1: Abb. 9, Bearbeitung 2: Abb. 10 und Abb. 11 sowie Bearbeitung 3: Abb. 12). Insgesamt hat jeweils eine Person aus jeder Gruppe zwar ein Beispiel angegeben, dieses aber nicht weiter genutzt, so dass für diese Personen nicht kodiert werden konnte, ob das Beispiel (bzw. die Selbsterklärungen) korrekt genutzt wurde. Insge- 
Tab. 3 Anzahl an hilfreichen Beispielen (bzw. Selbsterklärungen) je Darstellungsform

\begin{tabular}{lll}
\hline Hilfreich & & \\
& Graphisch & Symbolisch \\
\hline 3 (Bsp. ähnlich zu $\left.(-1)^{n} \cdot n\right)$ & 2 & 1 \\
2 (Bsp. ähnlich zu $(-1)^{n}$ ) & 3 & 1 \\
1 (Bsp. konstant oder monoton) & 18 & 13 \\
0 (Bsp. nicht hilfreich) & 13 & 14 \\
Insgesamt & 36 & 29 \\
\hline
\end{tabular}

Insgesamt 10 Studierende haben keine Aufzeichnungen angefertigt, die kodiert werden konnten

Tab. 4 Anzahl an korrekten Beispielen (bzw. Selbsterklärungen) je Darstellungsform

\begin{tabular}{lll}
\hline Korrekt & & \\
& Graphisch & Symbolisch \\
\hline 1 Punkt & 10 & 6 \\
0,5 Punkte & 7 & 4 \\
0 Punkte & 18 & 18 \\
Insgesamt & 35 & 28 \\
\hline
\end{tabular}

Zwei weitere Bearbeitungen konnten hier nicht kodiert werden, da die Studierenden zu dem genannten Beispiel keine weiteren Aufzeichnungen angefertigt hatten

samt zeigt sich, dass die meisten Studierenden kein korrektes und kein hilfreiches Beispiel konstruiert haben. Dies könnte ein Hinweis darauf sein, warum die konstruktive Bedingung der passiven Bedingung der Beispielnutzung nicht überlegen war, und liefert einen Einblick in die Nutzung von Beispielen zur Beweisillustration von Studierenden.

\section{Diskussion}

Beweise zu verstehen, ist eine wichtige Aktivität im Mathematikstudium, jedoch bereitet diese Aktivität vielen Studierenden große Schwierigkeiten (z. B. Mejía-Ramos et al. 2012). Eine mögliche Strategie beim Beweisverstehen, die in der Literatur diskutiert wird, ist das Illustrieren am Beispiel (Weber 2015; Wilkerson-Jerde und Wilensky 2011). Diese Strategie wird sowohl in Studien mit Experten-Novizen-Vergleichen als auch direkt von Dozierenden als lernförderlich herausgestellt. Unklar ist unserer Ansicht aber bisher, unter welchen spezifischen Bedingungen diese Strategie das Beweisverstehen tatsächlich fördert. Entgegen unseren Erwartungen konnten wir in dieser Studie mit einem Beweis aus dem Gebiet Analysis weder messbare Evidenzen dafür finden, dass graphische Darstellungsformen den symbolischen Darstellungsformen, noch, dass konstruktive Lernaktivitäten den passiven Lernaktivitäten beim Illustrieren am Beispiel überlegen sind. Dagegen repliziert diese Studie Erkenntnisse zu Prädiktoren von Beweisverständnis (vgl. Neuhaus und Rach 2019): Schulische Vorleistungen, gemessen durch die Gesamt-Abiturnote, und insbesondere inhaltliches Vorwissen bedingen das Beweisverständnis von Studierenden in der Studieneingangsphase. 
Dass die Faktoren „Darstellungsform“ und „Lernaktivität“ nicht die erwarteten Effekte auf das Beweisverständnis haben, kann durch die in der Literatur beschriebenen unterschiedlichen Wirkweisen erklärt werden. Beim Faktor ,Darstellungsform“ ist aus vor allem mathematikdidaktischer Sicht (z.B. Ostsieker 2020) ein grundsätzlicher Vorteil der Nutzung der graphischen Darstellungsform gegenüber der symbolischen Darstellungsform ableitbar, während die MultiMedia-Forschung (z.B. Schnotz und Bannert 2003) auch auf Nachteile der in einer Lernaktivität kombinierten Nutzung verschiedener Darstellungsformen hinweist. Bezüglich des Faktors „Lernaktivität“ könnte ein nicht-signifikanter Effekt in der Gesamtgruppe auch auf einen sogenannten expertise reversal effect (Kalyuga et al. 2003) in dem Sinne hindeuten, dass eine passive Lernaktivität möglicherweise besser für leistungsschwache Studierende geeignet ist, während eine konstruktive Lernaktivität für leistungsstarke Studierende einen besseren Erfolg bringt. Diese Vermutung müsste in weiterführenden Studien untersucht werden.

Durch die Analyse der Studierendenbearbeitungen haben wir weiterhin erste und für die weitere Forschungsarbeit wertvolle Hinweise erhalten, wie die Studierenden die Strategie der Beweisillustration nutzen und welche Art von Beispiel sie verwenden. Dabei konnten wir insbesondere beobachten, dass von den Studierenden der konstruktiven Gruppe zwar größtenteils Beispiele (und nur in einigen Fällen Selbsterklärungen statt Beispiele oder keines von beiden) entwickelt wurden, aber nur wenige Studierende korrekte und hilfreiche Beispiele entwickelt haben. Obwohl die passive Gruppe ein illustrierendes Beispiel auch für den zweiten Beweisfall zur Verfügung hatte, welches im Gegensatz zu den meisten der selbst entwickelten Beispiele in der konstruktiven Gruppe hilfreich und korrekt war, ist auch die passive der konstruktiven Gruppe im Beweisverständnistest nicht überlegen. Neben dem Einfluss des inhaltlichen Vorwissens auf den Bearbeitungsprozess bezieht sich ein weiterer Erklärungsansatz für dieses Phänomen auf die Art und Weise, in der die Studierenden das illustrierende Beispiel vorrangig nutzen (und zwar unabhängig davon, ob es gegeben war oder selbst konstruiert wurde). Die Bearbeitungsprozesse der konstruktiven Gruppen legen nahe, dass eine Reihe von Studierenden die Beispiele eher zur Illustration des Theorems und weniger zur Illustration der einzelnen Beweiszeilen nutzen: Sie wählen Beispielfolgen, die bereits offensichtlich das Theorem erfüllen, z. B. weil sie selbst schon monoton sind. In einem solchen Fall wird das im Beweis verwendete allgemeine Verfahren zur Auswahl einer monotonen Teilfolge einerseits nicht benötigt und würde andererseits einfach die Folge selbst liefern; als induktives Auswahlverfahren, das in jedem Fall eine eindeutige Teilfolge liefert, wird es also kaum deutlich. Demnach würden Beweis und Beispiel im Wesentlichen eher getrennt voneinander gelesen, ohne einen echten zeilenweisen Bezug herzustellen (in einigen Bearbeitungen scheint sich dies konkreter zu zeigen, indem von der allgemeinen Prozedur im Beweis im Beispiel abgewichen wird oder das Beispiel gar nicht zeilenweise weitergeführt wird). In Anlehnung an Ahmadpour et al. (2019) ließe sich diese Erklärhypothese auch so formulieren: Die Studierenden scheinen Beispiele eher im Rahmen eines semantischen Zugangs zum Theorem, nicht aber zum semantischen Beweisverstehen im Sinne der Konkretisierung der im Beweis verwendeten allgemeinen Prozeduren zu nutzen. Inwieweit sie den symbolischen, allgemeinen Beweis dabei eher syntaktisch (also als „formulaic proof“) verstehen, 
ist den Bearbeitungen nicht im Detail zu entnehmen; einzelne Bearbeitungen, in denen abstrakte Selbsterklärungsansätze statt Beispiele zur Verdeutlichung der einzelnen Beweisschritte gewählt wurden, lassen sich möglicherweise im Sinne eines stärker syntaktischen Zugangs zum Beweis deuten. Dieser zweite, theoretisch anknüpfungsfähige und mit den vorliegenden Daten konsistente Erklärungsansatz wäre insbesondere durch weiterführende empirische Studien zu prüfen. Das Studiendesign müsste dann ergänzend die Konstruktionsprozesse und -handlungen sowie die darin wirksamen Motive und Ziele der Studierenden in den Blick nehmen.

Unter Rückgriff auf Überlegungen aus Abschn. 2 dieses Beitrages schlagen wir in einem Ausblick Möglichkeiten im Sinne theoretischer und praktischer Implikationen vor, wie insbesondere das konstruktive Illustrieren am Beispiel als „Aufgabe an sich“ durch weitere, geeignete Maßnahmen stärker als in der vorgestellten Intervention gerahmt werden könnte, um lernförderlicher eingesetzt zu werden und beim Vergleich mit dem passiven Nachvollziehen eines illustrierenden Beispiels einen deutlich unterscheidbaren Effekt abzubilden.

Bevor wir solche Möglichkeiten anreißen, diskutieren wir Limitationen der Studie, die die Ergebnisse beeinflusst haben könnten und damit auch für die Bewertung der Studienergebnisse hinsichtlich der grundsätzlichen, lernförderlichen Einsetzbarkeit der Strategie relevant sind.

\subsection{Limitationen der Studie}

In dieser Studie wurden an einigen Stellen Entscheidungen getroffen, die im Kontext der Begriffe Verstehen-von-Beweisen und Verstehen-durch-Beweise (vgl. Abschn. 2.1) diskutiert werden können. Beispielsweise haben wir uns für ein spezielles, in der Literatur präsentes Modell entschieden, um Beweisverständnis zu messen, das sowohl Aspekte von Verstehen-von-Beweisen als auch von Verstehendurch-Beweise enthält. Nun stellt sich die Frage, ob bestimmte Varianten der Strategie „Illustrieren am Beispiel“ eher nur im Sinne einer der beiden Interpretationen von Beweisverständnis förderlich sind und somit die Auswirkungen der unterschiedlichen Varianten durch das Modell nicht adäquat abgebildet werden können. Zudem haben wir in dieser Studie Novizinnen und Novizen betrachtet, bei denen davon auszugehen ist, dass vorrangig Verstehen-von-Beweisen geschieht. Mehr oder weniger implizit haben wir dabei angenommen, dass es für diesen Personenkreis in jedem Fall hilfreich ist, Beweisschritte an einem Beispiel zu konkretisieren. Diese Annahme war möglicherweise falsch, es mag im Stadium eines vorrangigen Verstehens-von-Beweisen noch keine messbaren positiven Effekte geben (unabhängig von der Art der Beispielkonkretisierung, z. B. konstruktiv versus passiv bzw. graphisch versus symbolisch).

Im Sinne einer praktisch gut umsetzbaren und daher recht kurzen Intervention haben wir eine stark vorstrukturierte Darbietung der Strategie ,Illustrieren am Beispiel“" in Tabellenform entwickelt, um den zeilenweisen Bezug zwischen allgemeinem Beweisschritt (erste Spalte) und Beispielinstanz (zweite Spalte) zu verdeutlichen (vgl. Abb. 6). Für den ersten Fall der Fallunterscheidung im Beweis haben wir zudem den meisten Studierenden ein korrektes und hilfreiches Beispiel vorgelegt. Möglicherweise reicht diese Art der tabellarisch unterlegten Gegenüberstellung von 
Beweiszeile und konkreter Beispielinstanz für die Studierenden aber nicht aus, um eine semantische Verknüpfung zwischen den beiden Spalten aktiv herzustellen und nicht das Beispiel eher zur Illustration des bewiesenen Theorems zu verstehen.

Aufgrund von Zeitproblemen haben zudem nicht alle Studierenden den Vorwissenstest bearbeitet, so dass manche Analysen auf einer reduzierten Stichprobe basieren. Um ein Mediationsmodell von Vorwissen über den Bearbeitungsprozess zum Beweisverständnis zu rechnen, ist die gezogene Stichprobe in unseren Augen zu klein. Aber durch die Kodierung der Bearbeitungsprozesse in den konstruktiven Gruppen konnten wir explorative Überlegungen anstellen, wie das Vorwissen auf den Verstehensprozess wirkt, wie der Bearbeitungsprozess dann das Ergebnis des Verstehensprozesses beeinflusst und welche Arten von Beispielen die Studierenden nutzen (vgl. Abschn. 5.3).

Weiterhin ist unsere Studie nur im für die Studieneingangsphase besonders bedeutenden Gebiet der Analysis durchgeführt worden. Für geeignet gewählte Beweise in diesem Gebiet lassen sich verschiedene Darstellungsformen, insbesondere die graphische, zur Variation der hier untersuchten Unterstützungsmaßnahme sinnvoll nutzen; dies ist aber kein exklusives Argument für die Analysis. Es bleibt also die Frage bestehen, ob sich ähnliche Ergebnisse wie die hier vorgestellten auch in anderen mathematischen Gebieten, wie der Algebra und Zahlentheorie, finden lassen. Spezifische Beweisaktivitäten im Bereich der Analysis zu untersuchen, ist aber auch eine Stärke unserer Studie, denn die meisten anderen Studien zum Beweisverständnis sind bisher im Gebiet der Zahlentheorie durchgeführt worden (vgl. Mejía-Ramos und Weber 2020).

\subsection{Fazit und Ausblick auf praktische und theoretische Implikationen}

Die Ergebnisse unserer Studie zeigen dreierlei: Erstens hängt das Beweisverständnis eng mit dem konzeptuellen inhaltlichen Vorwissen zusammen. Zweitens konnte für keine der untersuchten Varianten der Strategie „Illustrieren am Beispiel“ ein klarer Vorteil gezeigt werden. Drittens unterscheidet sich die Nutzung der Strategie „Illustrieren am Beispiel“ durch die Studierenden zumindest unter Einschränkung auf die konstruktive Bedingung erheblich, sowohl in Bezug auf die Güte der selbst konstruierten Beispiele als auch darauf, wie konsequent eine Verknüpfung zwischen Beispielinstanz und Beweiszeile hergestellt wurde.

Die erste Erkenntnis führt zu der folgenden, in erster Linie praktischen, Implikation: Da Beweise vor allem in Vorlesungen von Dozierenden präsentiert und von Studierenden verstanden werden sollen, ist die Verknüpfung von konzeptuellem inhaltlichen Wissen und Beweisverständnis insbesondere in dieser Veranstaltungsform sinnvoll. Daher scheint es wichtig, zeitökonomische und zugleich effektive Möglichkeiten zu finden, um die benötigten Definitionen, Eigenschaften und Vorstellungen zu wichtigen Begriffen vor (oder während) der Präsentation eines Beweises zu wiederholen.

Als mögliche weitere, praktische wie auch theoretische Implikation führen wir die zweite und dritte Erkenntnis dieser Studie vor dem in Abschn. 2.4.1 kurz dargestellten Hintergrund zusammen. Demnach liegen auch aus anderen Studien (wie Iannone et al. 2011; Samkoff und Weber 2015) bereits Hinweise dafür vor, dass das 
eigentätige Illustrieren am Beispiel zur Unterstützung von Verstehen-durch-Beweise für Expertinnen und Experten besonders effektiv ist, Novizinnen und Novizen aber möglicherweise nicht per se durch das konstruktive Illustrieren an einem Beispiel im Verstehen-von-Beweisen stärker unterstützt werden als durch das bloße Nachvollziehen gegebener Beispiele. Die von uns in Anlehnung an die in Iannone et al. (2011) diskutierten Implikationen eingebrachte indirekte Instruktion zum Generieren hilfreicher Beispiele in Form eines für den ersten Beweisabschnitt (Fall 1) in allen Bedingungen vorgegebenen hilfreichen Beispiels mag dabei im Sinne einer ,explicit instruction in how to generate examples“ (ebd., S. 11) noch nicht hinreichend gewesen sein. In diesem Sinne schlagen wir hier vor, Studierende zunächst, zusätzlich zu einem einzelnen vorgegebenen hilfreichen Beispiel, stärker mit der Strategie des Illustrierens von Beweisschritten am Beispiel selbst vertraut zu machen, um sie dann in konkreten, konstruktiven Anwendungsfällen effektiver nutzen zu können. So können etwa die Bedingungen an ein hilfreiches und korrektes Beispiel zur Illustration von allgemeinen Prozeduren in Beweisschritten für die Studierenden expliziert und so besser sichtbar gemacht werden. Studierende können dabei exemplarisch vergleichende Betrachtungen von mehr und von weniger hilfreichen und korrekten illustrierenden Beispielen vornehmen. Wie weiter oben bereits angesprochen, sind weiterführende Studien z. B. im Mixed-Methods-Design nötig, die den Fokus stärker auf den jeweiligen Prozess der Beispielkonstruktion durch die Studierenden sowie auf den aus Sicht der Studierenden dabei tatsächlich illustrierten Gegenstand und die verwendeten Gütekriterien legen, um diese Überlegungen weiter zu stützen.

Beide Implikationen ließen sich dabei sinnvoll in einer Maßnahme kombinieren. Zwei konkrete Formate für eine mögliche Kombination sind: (1) Selbsterklärungsprompts als Einzelaktivität (vgl. Hodds et al. 2014); (2) Peer Instruction in einem kooperativen Setting. Selbsterklärungsprompts dienen der Aufforderung an Lernende, sich mit spezifischen Teilen von Texten oder Beweisen auseinander zu setzen (Hilbert et al. 2008). Die Lernenden können sowohl dazu aufgefordert werden, die einzelnen Schritte des Beweises mit ihrem inhaltlichen Vorwissen, insbesondere zu den verwendeten Begriffen, zu verbinden, als auch einzelne Schritte im Beweis mit einem möglichst hilfreichen Beispiel zu verknüpfen. Unter den kooperativen Lehrmethoden bietet sich beispielsweise Peer Instruction (Mazur 1997; Riegler 2020) als Möglichkeit an. In der speziellen Ausprägung als Mid-Proof Peer Instruction (Bauer und Skill 2019) kann sie inmitten eines laufenden Beweises eingesetzt werden, um benötigte Konzepte in konkretem Bezug zu ihrer Verwendung im Beweis zu aktivieren. Die Methode eignet sich auch, um Studierende zur Diskussion über den Bezug von konkreten Beispielen zu einzelnen Beweisschritten und über die Güte der Beispiele (insbesondere im Sinne von „hilfreich“ und „korrekt“) anzuregen.

Insgesamt wird deutlich, dass das Beweisverstehen ein wichtiger und herausfordernder Prozess in der Studieneingangsphase eines Mathematikstudiums ist. Um Beweise zu verstehen, ist ein adäquates, inhaltliches Vorwissen notwendig. Neben dem inhaltlichen Vorwissen erscheint der explizite Aufbau eines grundlegenden Repertoires an Strategien, die den Verstehensprozess unterstützen können, essentiell. Welche Strategien sich hierfür am besten eignen und unter welchen spezifischen Bedingungen und im Rahmen welcher konkreten Unterstützungsformate diese Strategien lernförderlich wirksam werden, sind wichtige, weiter zu untersuchende Fragen. 


\section{Anhang A}

\section{Weitere Items aus dem Beweisverständnistest}

Beispiel für die lokale Ebene

Zu Schritt (6): Ein solches $k_{2}$ existiert, ...

$\square$... weil $k_{1}$ sonst eine Gipfelstelle der Folge $\left(a_{n}\right)$ wäre.

$\square \ldots$ weil $k_{1}$ in Schritt (4) als kleinste solche Stelle gewählt wurde.

$\square \ldots$ weil die Folge $\left(a_{n}\right)$ monoton steigend ist.

$\square$... weil die Menge der Indizes $n$ mit $a_{n} \geq a_{k_{1}}$ ein Minimum besitzt.

Abb. 7 Beispielitem aus dem Beweisverständnistest zum lokalen Beweisverständnis (5) 


\section{Beispiel für die ganzheitliche Ebene}

Den Beweis kann man in drei inhaltlich zusammenhängende Teilabschnitte zerlegen. Geben Sie eine mögliche solche Zerlegung mit Hilfe der Schrittnummern (1)-(8) an und benennen Sie die Abschnitte inhaltlich passend mit Überschriften.

Zerlegung:

Teil 1, Nummern: Überschrift:

Teil 2, Nummern: Überschrift:

Teil 3, Nummern: Überschrift:

Abb. 8 Beispielitem aus dem Beweisverständnistest zum ganzheitlichen Beweisverständnis (6) 


\section{Anhang B}

\section{Beispielkonstruktionen von Studierenden}

Zur Verdeutlichung des Kodierungsmanuals zeigen wir hier Ausschnitte aus den Beispielkonstruktionen von Studierenden aus der graphisch-konstruktiven Gruppe. Für die Zwecke der Intervention war der Beweis in acht Einzelschritte gegliedert. Wie in Abb. 6 gezeigt, waren die ersten beiden Schritte durch ein vorgegebenes Beispiel illustriert, gefolgt von der Aufforderung, sich auch die weiteren sechs Argumentationsschritte des Beweises in ähnlicher Weise zu verdeutlichen.

Bearbeitung 1 (hilfreich: 3, korrekt: 0)

Das Bild zeigt, wie eine Studierende oder ein Studierender Schritt (3) des Beweises durch eine alternierend wachsende Folge in graphischer Darstellung illustriert. Gemäß dem in Abschn. 4.6 erläuterten Kodierschema wurde dieses Beispiel bezüglich Aspekt 1 als sehr hilfreich (Code 3) eingeordnet - es handelt sich um ein Beispiel, wie es auch Expertinnen und Experten wählen würden. Der Studierende verfolgt das Beispiel allerdings in den nächsten Beweisschritten nicht weiter, so dass die Bearbeitung unter Aspekt 2 (Korrektheit des Umgangs mit dem Beispiel) unter Code 0 eingeordnet wurde.

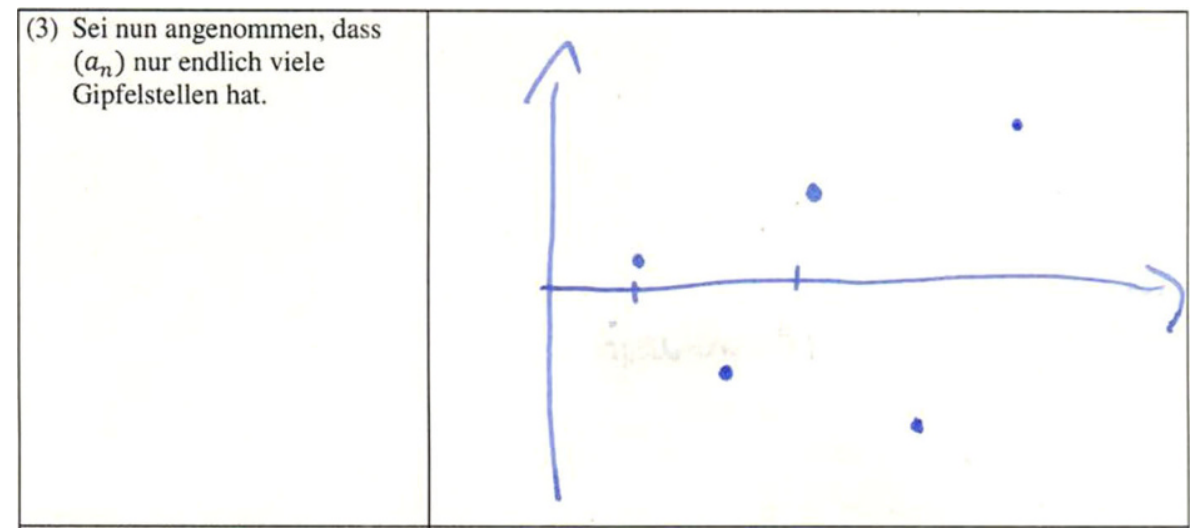

Abb. 9 Beispielkonstruktion Bearbeitung 1: Schritt 3 
Bearbeitung 2 (hilfreich: 2, korrekt: 0.5)

Diese bzw. dieser Studierende gibt zu Schritt (3) eine Folge an, die schließlich (d.h. ab einem gewissen Index) zwischen zwei konstanten Folgen alterniert. Unter dem Aspekt 1 wurde dies als hilfreich (Code 2) eingeordnet. Eine solche Beispielfolge ist insofern hilfreich, als das im Beweis gezeigte Vorgehen eine echte Teilfolge auswählt. Da diese konstant ist, stellt das Beispiel allerdings einen Spezialfall dar und engt die Sicht auf den Beweis möglicherweise ein.

Die weitere Verarbeitung des Beispiels (Aspekt 2) wurde als teilweise korrekt (Code 0,5) eingestuft. So ist etwa in Schritt (7) der Index $k_{3}$ nicht passend zum Beweis gewählt (das Bild zeigt nicht den kleinsten solchen Index). Würde eine Teilfolge auf diese Weise ausgewählt, so entstünde zwar eine monotone Teilfolge, aber nicht dieselbe, die das Vorgehen im Beweis erzeugt. Die Beispielverarbeitung weicht insofern vom Vorgehen im Beweis ab.

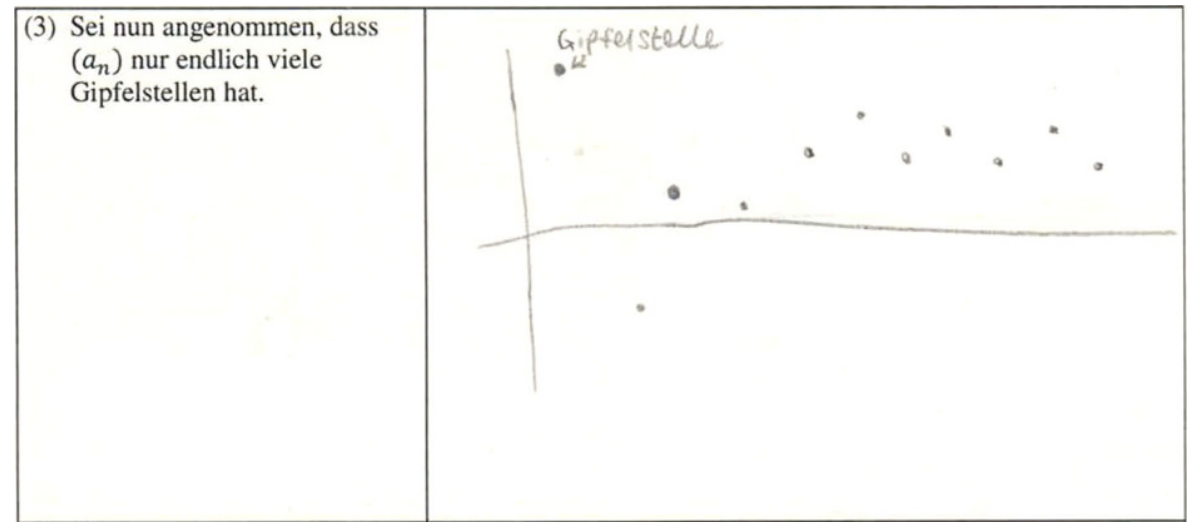

Abb. 10 Beispielkonstruktion Bearbeitung 2: Schritt 3

(7) Da auch $k_{2}$ keine Gipfelstelle ist, existiert ein $k_{3}>k_{2}$ mit $a_{k_{3}} \geq a_{k_{2}}$. Sei $k_{3}$ die kleinste solche Stelle.

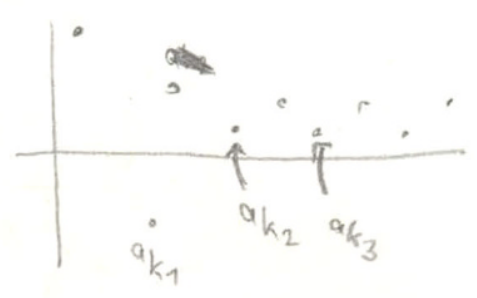

Abb. 11 Beispielkonstruktion Bearbeitung 2: Schritt 7 
Bearbeitung 3 (hilfreich: 0, korrekt: 0)

Die Bearbeitung dieser oder dieses Studierenden zeigt zweierlei: Zum einen legt sie oder er zu Schritt (3) ein Koordinatensystem an, in dem eine Beispielfolge eingezeichnet werden könnte, wechselt aber stattdessen zu einer Selbsterklärung in algebraischer Darstellung. Zum anderen zieht sie oder er (irrtümlich) den Schluss, dass die Folge schließlich konstant sein müsse, wenn sie nur endlich viele Gipfelstellen hat. Da weder ein hilfreiches Beispiel noch eine hilfreiche Selbsterklärung gebildet wurde, ist diese Bearbeitung mit Code 0 versehen.
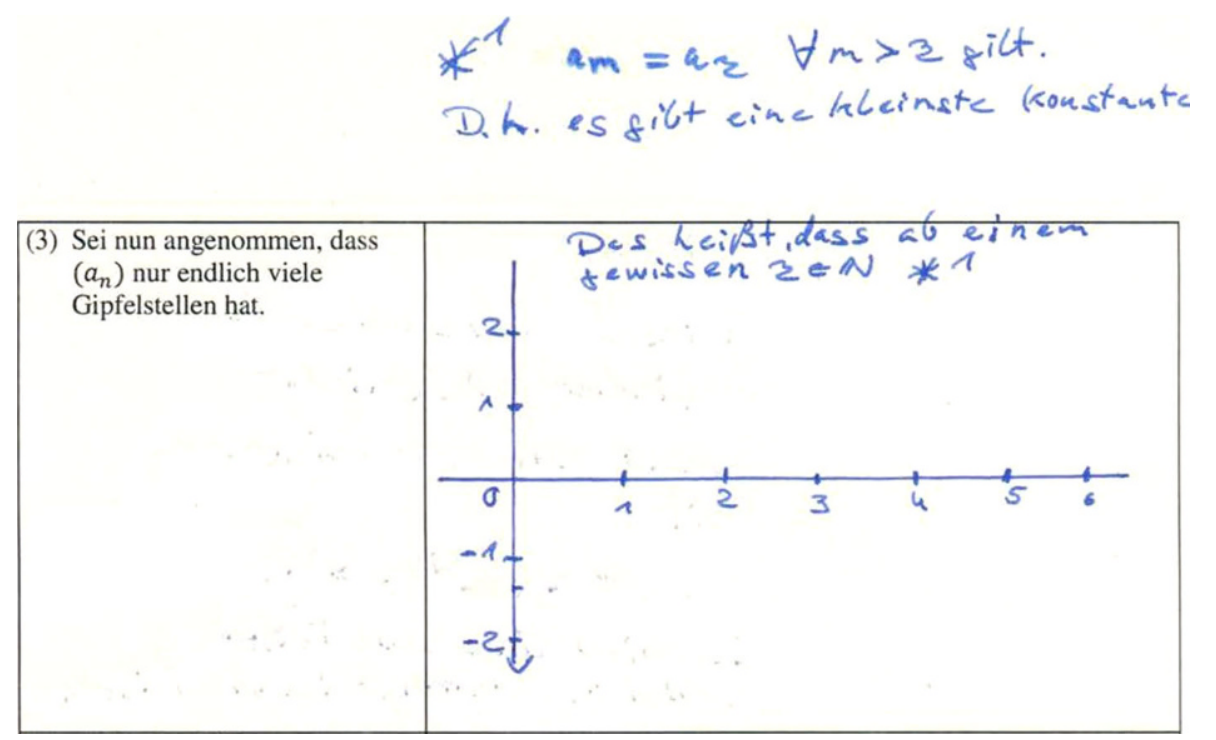

Abb. 12 Beispielkonstruktion Bearbeitung 3: Schritt 3 
Funding Open Access funding enabled and organized by Projekt DEAL.

Open Access Dieser Artikel wird unter der Creative Commons Namensnennung 4.0 International Lizenz veröffentlicht, welche die Nutzung, Vervielfältigung, Bearbeitung, Verbreitung und Wiedergabe in jeglichem Medium und Format erlaubt, sofern Sie den/die ursprünglichen Autor(en) und die Quelle ordnungsgemäß nennen, einen Link zur Creative Commons Lizenz beifügen und angeben, ob Änderungen vorgenommen wurden.

Die in diesem Artikel enthaltenen Bilder und sonstiges Drittmaterial unterliegen ebenfalls der genannten Creative Commons Lizenz, sofern sich aus der Abbildungslegende nichts anderes ergibt. Sofern das betreffende Material nicht unter der genannten Creative Commons Lizenz steht und die betreffende Handlung nicht nach gesetzlichen Vorschriften erlaubt ist, ist für die oben aufgeführten Weiterverwendungen des Materials die Einwilligung des jeweiligen Rechteinhabers einzuholen.

Weitere Details zur Lizenz entnehmen Sie bitte der Lizenzinformation auf http://creativecommons.org/ licenses/by/4.0/deed.de.

\section{Literatur}

Acevedo Nistal, A., van Dooren, W., \& Verschaffel, L. (2014). Improving students' representational flexibility in linear-function problems: an intervention. Educational Psychology, 34(6), 763-786.

Ahmadpour, F., Reid, D., \& Fadaee, M. R. (2019). Students' ways of understanding a proof. Mathematical Thinking and Learning, 21(2), 85-104.

Balacheff, N. (1988). Aspects of proof in pupils' practice of school mathematics. In D. Pimm (Hrsg.), Mathematics, teachers and children (S. 216-235). London: Hodder and Stoughton.

Bauer, T., \& Skill, T. (2019). Peer-Instruction zur Förderung des Beweisverständnisses in mathematischen Vorlesungen. In A. Meissner, C. Walter, B. Zinger \& J. Haubner (Hrsg.), Tagungsband zum 4. MINTSymposium zur Hochschullehre in den MINT-Fächern (S. 38-46). Nürnberg: TH Nürnberg.

Biehler, R., \& Kempen, L. (2016). Didaktisch orientierte Beweiskonzepte - Eine Analyse zur mathematikdidaktischen Ideenentwicklung. Journal für Mathematik-Didaktik, 37, 141-179.

Bruner, J. (1971). Studien zur kognitiven Entwicklung. Stuttgart: Klett.

Brunner, E. (2014). Mathematisches Argumentieren, Begründen und Beweisen. Grundlagen, Befunde und Konzepte. Berlin, Heidelberg: Springer Spektrum.

Chi, M. T.H. (2009). Active-constructive-interactive: a conceptual framework for differentiating learning activities. Topics in cognitive science, 1(1), 73-105.

Chi, M. T.H., \& Wylie, R. (2014). The ICAP framework: linking cognitive engagement to active learning outcomes. Educational Psychologist, 49(4), 219-243.

Chi, M. T. H., Bassok, M., Lewis, M. W., Reimann, P., \& Glaser, R. (1989). Self-explanations: how students study and use examples in learning to solve problems. Cognitive Science, 13, 145-182.

Clark, M., \& Lovric, M. (2009). Understanding secondary-tertiary transition in mathematics. International Journal of Mathematical Education in Science and Technology, 40(6), 755-776.

Conradie, J., \& Frith, J. (2000). Comprehension tests in mathematics. Educational Studies in Mathematics, 42(3), 225-235.

Gagatsis, A., Elia, I., Panaoura, A., Gravvani, K., \& Spyrou, P. (2006). An empirical four-dimension model for the understanding of function. In J. Novotná (Hrsg.), Proceedings of the 30th Conference of the International Group for the Psychology of Mathematics Education (S. 137-144). Prague: PME.

Härtig, H., Fraser, N., Bernholt, S., \& Retelsdorf, J. (2019). Kann man Sachtexte vereinfachen? - Ergebnisse einer Generalisierungsstudie zum Textverständnis. ZfDN, 25(1), 273-287.

Heintz, B. (2000). Die Innenwelt der Mathematik. Zur Kultur und Praxis einer beweisenden Disziplin. Wien, New York: Springer.

Heuser, H. (1980). Lehrbuch der Analysis, Teil 1. Stuttgart: Teubner.

Hilbert, T. S., Renkl, A., Kessler, S., \& Reiss, K. (2008). Learning to prove in geometry: learning from heuristic examples and how it can be supported. Learning and Instruction, 18, 54-65.

Hodds, M., Alcock, L., \& Inglis, M. (2014). Self-explanation training improves proof comprehension. Journal for Research in Mathematics Education, 45(1), 62-101. 
Iannone, P., Inglis, M., Mejía-Ramos, J. P., Simpson, A., \& Weber, K. (2011). Does generating examples aid proof production? Educational Studies in Mathematics, 77, 1-14.

Inglis, M., \& Alcock, L. (2012). Expert and novice approaches to reading mathematical proofs. Journal for Research in Mathematics Education, 43(4), 358-390.

Kalyuga, S., Ayres, P., Chandler, P., \& Sweller, J. (2003). The expertise reversal effect. Educational Psychologist, 38(1), 23-31.

Kirsch, A. (1979). Beispiele für prämathematische Beweise. In W. Dörfler \& R. Fischer (Hrsg.), Beweisen im Mathematikunterricht (S. 261-274). Wien: Hölder-Pichler-Tempsky.

Klein, F. (1908). Arithmetik, Algebra und Analysis. Bd. I. Berlin: Springer.

Krantz, S. G. (1999). How to teach mathematics. Providence: American Mathematical Society.

Leppink, J., Broers, N. J., Imbos, T., van der Vleuten, C. P. M., \& Berger, M. P.F. (2012). Self-explanation in the domain of statistics: an expertise reversal effect. Higher Education, 63, 771-785.

Leron, U. (1983). Structuring mathematical proofs. The American Mathematical Monthly, 90(3), $174-185$.

Mali, A. (2014). Lecturers' use of generic examples: the generic set. Research in Mathematics Education, 16(3), 324-325.

Mazur, E. (1997). Peer instruction: getting students to think in class. In E. F. Redish \& J. S. Rigden (Hrsg.), The changing role of physics departments in modern universities. AIP Conference Proceedings 399 (S. 981-988).

Mejía-Ramos, J.P., \& Weber, K. (2020). Using task-based interviews to generate hypotheses about mathematical practice: mathematics education research on mathematicians' use of examples in proofrelated activities. ZDM Mathematics Education, 52, 1063-1074.

Mejía-Ramos, J.P., Fuller, E., Weber, K., Rhoads, K., \& Samkoff, A. (2012). An assessment model for proof comprehension in undergraduate mathematics. Educational Studies in Mathematics, 79(1), 3-18.

Mills, M. (2014). A framework for example usage in proof presentations. The Journal of Mathematical Behavior, 33, 106-118.

Müller, G., \& Wittmann, E. (1988). Wann ist ein Beweis ein Beweis? In P. Bender (Hrsg.), Mathematikdidaktik - Theorie und Praxis. Festschrift für Heinrich Winter (S. 237-258). Berlin: Cornelsen.

Müller-Hill, E. (2011). Die epistemische Rolle formalisierbarer mathematischer Beweise - Formalisierbarkeitsorientierte Konzeptionen mathematischen Wissens und mathematischer Rechtfertigung innerhalb einer sozio-empirisch informierten Erkenntnistheorie der Mathematik. https://bonndoc.ulb.unibonn.de/xmlui/bitstream/handle/20.500.11811/4850/2526.pdf?sequence=1\&isAllowed=y. Zugegriffen: 24. Sept. 2020.

Müller-Hill, E. (2017). Eine handlungsorientierte didaktische Konzeption nomischer mathematischer Erklärung. Journal für Mathematik-Didaktik, 38, 167-208.

Neuhaus, S., \& Rach, S. (2019). Proof comprehension of undergraduate students and the relation to individual characteristics. In U.T. Jankvist, M. Van den Heuvel-Panhuizen \& M. Veldhuis (Hrsg.), Proceedings of the eleventh congress of the European Society for Research in Mathematics Education. Utrecht: Freudenthal Group \& Freudenthal Institute, Utrecht University and ERME.

Ostsieker, L. (2020). Lernumgebungen für Studierende zur Nacherfindung des Konvergenzbegriffs: Gestaltung und empirische Untersuchung. Berlin: Springer.

Pracht, E. (1979). Beweisverständnis und dessen Überprüfbarkeit. In W. Dörfler \& R. Fischer (Hrsg.), Beweisen im Mathematikunterricht. Schriftenreihe Didaktik der Mathematik, (Bd. 2, S. 349-356). : Hölder-Pichler-Tempsky.

Rach, S. (2014). Charakteristika von Lehr-Lern-Prozessen im Mathematikstudium: Bedingungsfaktoren für den Studienerfolg im ersten Semester. Münster, New York: Waxmann.

Rav, Y. (1999). Why do we prove theorems? Philosophia mathematica, 7(1), 5-41.

Riegler, P. (2020). Peer Instruction in der Mathematik: Didaktische, organisatorische und technische Grundlagen praxisnah erläutert. Berlin: Springer.

Rittle-Johnson, B., Loehr, A. M., \& Durkin, K. (2017). Promoting self-explanation to improve mathematics learning: a meta-analysis and instructional design principles. ZDM Mathematics Education, 49, 599-611.

Roh, K.H. (2009). Students' understanding and use of logic in evaluation of proofs about convergence. In F.-L. Lin, F.-J. Hsieh, G. Hanna \& M. de Villiers (Hrsg.), Proceedings of the ICMI study 19 conference: proof and proving in mathematics education. The Department of Mathematics, National Taiwan Normal University. (S. 148-153).

Ronda, E. (2015). Growth points in linking representations of function: a research-based framework. Educational Studies in Mathematics, 90, 303-319. 
Rosseel, Y. (2012). Lavaan: An R package for structural equation modeling. Journal of Statistical Software, 2, 1-36.

Rowland, T. (2002). Generic proofs in number theory. In S. R. Campbell \& R. Zazkis (Hrsg.), Learning and teaching number theory. Research in cognition and instruction (S. 157-183). Westport: Ablex.

Roy, S., Alcock, L., \& Inglis, M. (2010). Undergraduates' proof comprehension: A comparative study of three forms of proof presentation. Proceedings of the 13th Conference on Research in Undergraduate Mathematics Education. http://sigmaa.maa.org/rume/crume2010/Archive/Roy\%20et\%20al.pdf. Zugegriffen: 28. Sept. 2020.

Roy, S., Inglis, M., \& Alcock, L. (2017). Multimedia resources designed to support learning from written proofs: an eye-movement study. Educational Studies in Mathematics, 96(2), 249-266.

Samkoff, A., \& Weber, K. (2015). Lessons learned from an instructional intervention on proof comprehension. The Journal of Mathematical Behavior, 39, 28-50.

Schnotz, W., \& Bannert, M. (2003). Construction and interference in learning from multiple representations. Learning and Instruction, 13, 141-156.

Selden, A. (2012). Transitions and proof and proving at tertiary level. In G. Hanna \& M. de Villiers (Hrsg.), Proof and proving in mathematics education: the 19th ICMI study (S. 391-422). Heidelberg: Springer.

Semadeni (1974). The concept of premathematics as a theoretical background for primary mathematics teaching. Warsaw: Polish Academy of Sciences.

Sommerhoff, D. (2017). The individual cognitive resources underlying students' mathematical argumentation and proof skills. Dissertation. Ludwig-Maximilians-Universität.

Tall, D. (2008). The transition to formal thinking in mathematics. Mathematics Education Research Journal, 20(2), 5-24.

Tall, D., \& Vinner, S. (1981). Concept image and concept definition in mathematics with particular reference to limits and continuity. Educational Studies in Mathematics, 12(2), 151-169.

Trapmann, S., Hell, B., Weigand, S., \& Schuler, H. (2007). Die Validität von Schulnoten zur Vorhersage des Studienerfolgs - eine Metaanalyse. Zeitschrift für Pädagogische Psychologie, 21(1), 11-27.

Ufer, S. (2015). The role of study motives and learning activities for success in first semester mathematics studies. In K. Beswick, T. Muir \& J. Wells (Hrsg.), Proceedings of the 39th conference of the international group for the psychology of mathematics education (S. 265-272). Hobart: PME.

De Villiers, M. (1990). The role and function of proof in mathematics. Pythagoras, 24(1), 17-24.

Watson, A., \& Mason, J. (2005). Mathematics as a constructive activity: Learners generating examples. Mahwah: Erlbaum.

Weber, K. (2001). Student difficulty in constructing proofs: the need for strategic knowledge. Educational Studies in Mathematics, 48, 101-119.

Weber, K. (2002). Beyond proving and explaining: proofs that justify the use of definitions and axiomatic structures and proofs that illustrate technique. For the learning of mathematics, 22(3), 14-17.

Weber, K. (2012). Mathematicians' perspectives on their pedagogical practice with respect to proof. International Journal of Mathematical Education in Science and Technology, 43(4), 463-482.

Weber, K. (2015). Effective proof reading strategies for comprehending mathematical proofs. International Journal of Research in Undergraduate Mathematics Education, 1(3), 289-314.

Weber, K., \& Mejía-Ramos, J.P. (2011). Why and how mathematicians read proofs: an exploratory study. Educational Studies in Mathematics, 76(3), 329-344.

Weber, K., \& Mejía-Ramos, J.P. (2013). Effective but underused strategies for proof comprehension. In M. Martinez \& A. Castro Superfine (Hrsg.), Proceedings of the 35th annual meeting of the North American Chapter of the International Group for the Psychology of Mathematics Education (S. 260-267). Chicago: University of Illinois at Chicago.

Wilkerson-Jerde, M.H., \& Wilensky, U.J. (2011). How do mathematicians learn math? resources and acts for constructing and understanding mathematics. Educational Studies in Mathematics, 78(1), 21-43.

Zaslavsky, O. (2019). There is more to examples than meets the eye: Thinking with and through mathematical examples in different settings. The Journal of Mathematical Behavior, 53, 245-255. 